\title{
Moving Bed Granular Bed Filter Development Program
}

\section{Topical Report \\ September 1994}

By:

J.C. Haas

J.W. Prudhomme

K. W. Wilson

$$
\begin{aligned}
& \text { DEOEIVED } \\
& \text { OCT } 141997 \\
& \text { OSTI }
\end{aligned}
$$

Work Performed Under Contract No.: DE-AC21-90MC27423

For

U.S. Department of Energy

Office of Fossil Energy

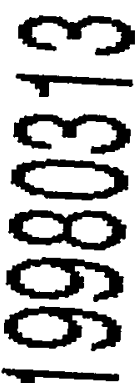

Federal Energy Technology Center

Morgantown Site

Morgantown, West Virginia

By

Combustion Power Company

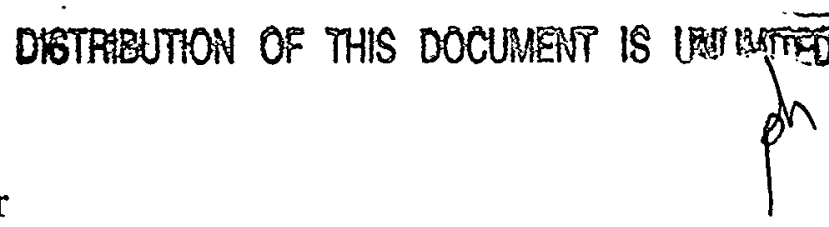

Oakland, California

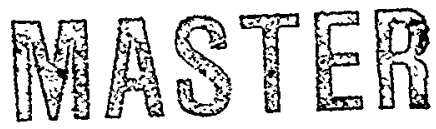




\section{DISCLAIMER}

This report was prepared as an account of work sponsored by an agency of the United States Government. Neither the United States Government nor any agency thereof, nor any of their employees, makes any warranty, express or implied, or assumes any legal liability or responsibility for the accuracy, completeness, or usefulness of any information, apparatus, product, or process disclosed, or represents that its use would not infringe privately owned rights. Reference herein to any specific commercial product, process, or service by trade name, trademark, manufacturer, or otherwise does not necessarily constitute or imply its endorsement, recommendation, or favoring by the United States Government or any agency thereof. The views and opinions of authors expressed herein do not necessarily state or reflect those of the United States Government or any agency thereof.

Available to the public from the National Technical Information Service, U.S. Department of Commerce, 5285 Port Royal Road, Springfield, VA 22161; phone orders accepted at (703) 487-4650. 


\section{DISCLAIMER}

\section{Portions of this document may be illegible in electronic image products. Images are produced from the best available original document.}




\title{
Moving Bed Granular Bed Filter Development Program
}

\author{
Topical Report \\ September 1994
}

\author{
By: \\ J.C. Hass \\ J.W. Prudhomme \\ K.W. Wilson
}

Work Performed Under Contract No.: DE-AC21-90MC27423

\author{
For \\ U.S. Department of Energy \\ Office of Fossil Energy \\ Federal Energy Technology Center \\ Morgantown Site \\ P.O. Box 880 \\ Morgantown, West Virginia 26507-0880
}

By

Combustion Power Company

201 Webster Street

Suite 1700

Oakland, California 94612 
TABLE OF CONTENTS

1.0 INTRODUCTION

2.0 DESCRIPTION OF PROCESS CONTROLS AND INSTRUMENTATION

3.0 DESCRIPTION OF SAMPLING AND ANALYTICAL EQUIPMENT

4.0 EQUTPMENT SPECIFICATIONS

5.0 INSTRUMENT AND VALVE AND SPECIFICATIONS

6.0 UTILITY REQUIREMENTS

7.0 ESTIMATED COSTS

8.0 SCHEDUIE

9.0 ENGINEERING DRAWINGS

Granular Bed Test Filter Process Flow Diagram

Granular Bed Test Filter Piping and Instrument Diagram Granular Bed Test Filter General Arrangement

Granular Bed Test Filter Detail Drawings Support structure Split Filter Full Size Filters $-3.6 \mathrm{ft}$ and $6.0 \mathrm{ft}$ Diameter Media Fluidizer Test Unit Lift Pipe Liner Abrasion Test Unit 


\subsection{INTRODUCTION}

Five test arrangements have been designed to support the Granular Bed Filter Development Program as defined in the Test Plan described in Task 3. The first arrangement is a $3.6 \mathrm{ft}$ diameter half filter, with a glass covering along the cross section to allow visual examination of the granular alumina material passing through the filter. This unit will be used to evaluate up to 4 different filter discharge hopper designs and two different air inlet pipe configurations.

The second test arrangement is a $3.6 \mathrm{ft}$ diameter full size filter having refractory lining to simulate actual surface roughness conditions. Results from the half filter testing will be applied to the hopper and inlet pipe design and positioning on the full size filter.

The third test arrangement will examine filter geometry scale up by testing a $6.0 \mathrm{ft}$ diameter full size filter. The $6.0 \mathrm{ft}$ filter will also have refractory lining to simulate actual surface roughness. All three of the filter units will be supported from a common structure and will use a common pneumatic transport system to circulate alumina from the filter drain back to the filter top.

The forth Test Arrangement consists of a small 12 inch diameter fluidizer to measure the minimum fluidization velocity of the $7 \mathrm{~mm}$ (approximate size) alumina material to be used in the filter assemblies.

The last Test Unit is used to evaluate relative abrasion characteristics of potential refractory and ceramic materials to be installed in high abrasion areas in the pneumatic transport piping. The unit consists of several 40" diameter cylinders each having a liner sample and alumina material. Each revolution alumina falls on to the liner surface causing some level of abrasion. The test is designed to run for $1000 \mathrm{hrs}$. 


\subsection{DESCRIPTION OF PROCESS CONTROLS}

\subsection{Granular Bed Filter Controls}

The instrumentation and valving for the granular bed filter test units are defined on the Piping and Instrument Diagram included in this report. Air is supplied to the filter inlet using one or two blowers, depending on the filter size and capacity. Air flow is controlled manually with butterfly valves at each blower inlet. Flow is measured using an orifice.

For some of the planned tests, ash is introduced into the inlet air stream to determine the effects of ash on alumina material flow characteristics. The ash is from a local coal fired power plant and is metered into the air stream using a small bin with a variable speed, screw conveyor. An eductor is used to overcome the inlet air pressure and pneumatically convey the ash to the filter inlet pipe.

Inside the filter, the ash is captured by the granular alumina material and clean air exits the filter top. The test arrangement is located outdoors so the filter top is open to atmosphere. The filter material and ash drain out the filter bottom through a seal leg to a pneumatic lift pipe to return the alumina back to the filter top for another cycle. During the pneumatic transport process, ash is dislodged from the alumina and the ash exits with the lift air at the deentrainment Vessel, due to the size and density difference. Instrumentation is positioned on the filter to manually record filter pressure drop, filter seal leg pressure drops, Iift pipe pressure drop and de-entrainment vessel pressure. The filter pressure drop versus filter air flow are important performance measurements. The seal leg pressure drop is used to confirm that air is exiting from the filter rather than entering the filter at the seal leg. The out flow of air at the seal leg is necessary to maintain proper ash flow draining from the filter. A bleed line is mounted from the seal leg to the ash collection baghouse to maintain air flow down the seal leg when lift pipe air pressure is higher than the filter pressure.

The alumina circulation rate is controlled by varying the amount of injection air on the "L- Valve" at the base of the seal leg. The alumina flow rate is proportional to the lift pipe pressure drop and is used to set the circulation rate. A sight glass is mounted on the seal leg to visually measure the alumina velocity which is used to confirm the material mass flow. The lift air is supplied by a separate positive displacement type blower. The lift air flow is established by venting excess air at the blower discharge because the blower has fixed speed. 
In addition to filter pressure drop, the filter flow characteristics will be determined by measuring the air flow distribution at the filter top using a thermoanemometer (designed for low air velocities). The alumina flow distribution will be determined by the movement of metal rods which descend into the filter with the alumina granules. The 3.6 ft diameter half filter will also allow visual observation of the alumina flow profile.

From the de-entrainment vessel, ash is pneumatically transported to a baghouse where the ash is separated from the air. The ash particles drain from the baghouse hopper to a 55 galion drum for reuse or disposal. A suction fan is mounted downstream of the baghouse to control the de-entrainment vessel near atmospheric pressure. The fan is controlled manually by adjusting the inlet butterfly, similar to the air supply blowers.

\subsection{Alumina Fluidizer Controls}

The fluidizer unit is used to measure the minimum fluidization velocity of the $7 \mathrm{~mm}$ alumina material. Fluidizing air is supplied by one of the air supply blowers as described in section 2.1. The unit is operated by gradually increasing the air flow while measuring the pressure drop across the bed until the bed begins to fluidize. The pressure drop versus air flow data as well as visual observation determine when fluidization begins.

\subsection{Lift Pipe Liner Abrasion Unit Controls}

Candidate refractory and ceramic materials will be tested for abrasion using a rotating drum abrasion test unit. Each refractory sample will be mounted in a separate fabricated enclosed cylinder along with a defined amount of $7 \mathrm{~mm}$ alumina. With each revolution alumina will drop on the test sample surface at a velocity similar to that inside the filter pneumatic lift pipe.

Each sample will be fired to typical operating temperatures ( $\sim 1600 \mathrm{~F}$ ) and weighed before testing. The cylinders are then assembled together to form one drum which mounts on a standard drum rotator. The $1 / 2$ horsepower drum rotator operates at 10 RPM for 1000 Hours. Each refractory sample is divided into two sections to simplify casting and each weigh approximately 30 lbs, depending on the sample thickness and material. The dust and media will be collected and weighed for mass balance. 


\subsection{DESCRIPTION OF SAMPIING AND ANALYTICAL EQUIPMENT \\ - Toledo Digital Scale (0-100 1bs, accuracy to .01 1bs) \\ - Triple Beam Balance (0-2 kg, accuracy to .1 g)}

\subsection{EQUIPMENT BPECIFICATIONS}

ITEM

Air Supply Blower No.1

Air Supply Blower Mo.2

Ash Eductor

Ash Feeder

Granular Bed Filter

Bleed Eductor

Lift System PD Blower

Baghouse

Suction Fan

Air Compressor

Fluidizer

Abrasion Test Drums

Drum Rotator
MANUFACTURER STATUS CAPACITY

\begin{tabular}{|c|c|c|}
\hline Hoffman & Existing & 2570 ACFH at 6.3 PSIG \\
\hline Lamson or Eq. & Rental & 2100 ACFM at 6.3 PSIG \\
\hline CPC or Fox & Нен & $2^{\prime \prime}$ Dia \\
\hline Acrison or Eq. & Rental & $12 \mathrm{Lb} / \mathrm{Hr}$ \\
\hline $\mathrm{CPC}$ & Нен & 3.6 and $6.0 \mathrm{Ft} \mathrm{Dia}$ \\
\hline CPC or Fox & Кен & 1.5" Dia \\
\hline PEGO or Eq. & Rental & 260 ACFH at 6 PSIG \\
\hline Micropul & Existing & 900 ACFH at $6: 1$ Air/ Cloth \\
\hline Buffalo forge & Existing & 850 ACFH at 16 IWG static DP \\
\hline US Rental & Rental & 100 SCFM at 100 PSIG \\
\hline CPC & New & 12" Dia \\
\hline CPC & New & 40" Dia \\
\hline $\begin{array}{l}\text { Advanced } \\
\text { Handl ing sys. }\end{array}$ & Hew & 400 Lbs $\max$ at $10 \mathrm{RPM}$ \\
\hline
\end{tabular}




\subsection{INSTRUMENT AND VALVE SPECIFICATIONS}

\section{INSTRUMENTS :}

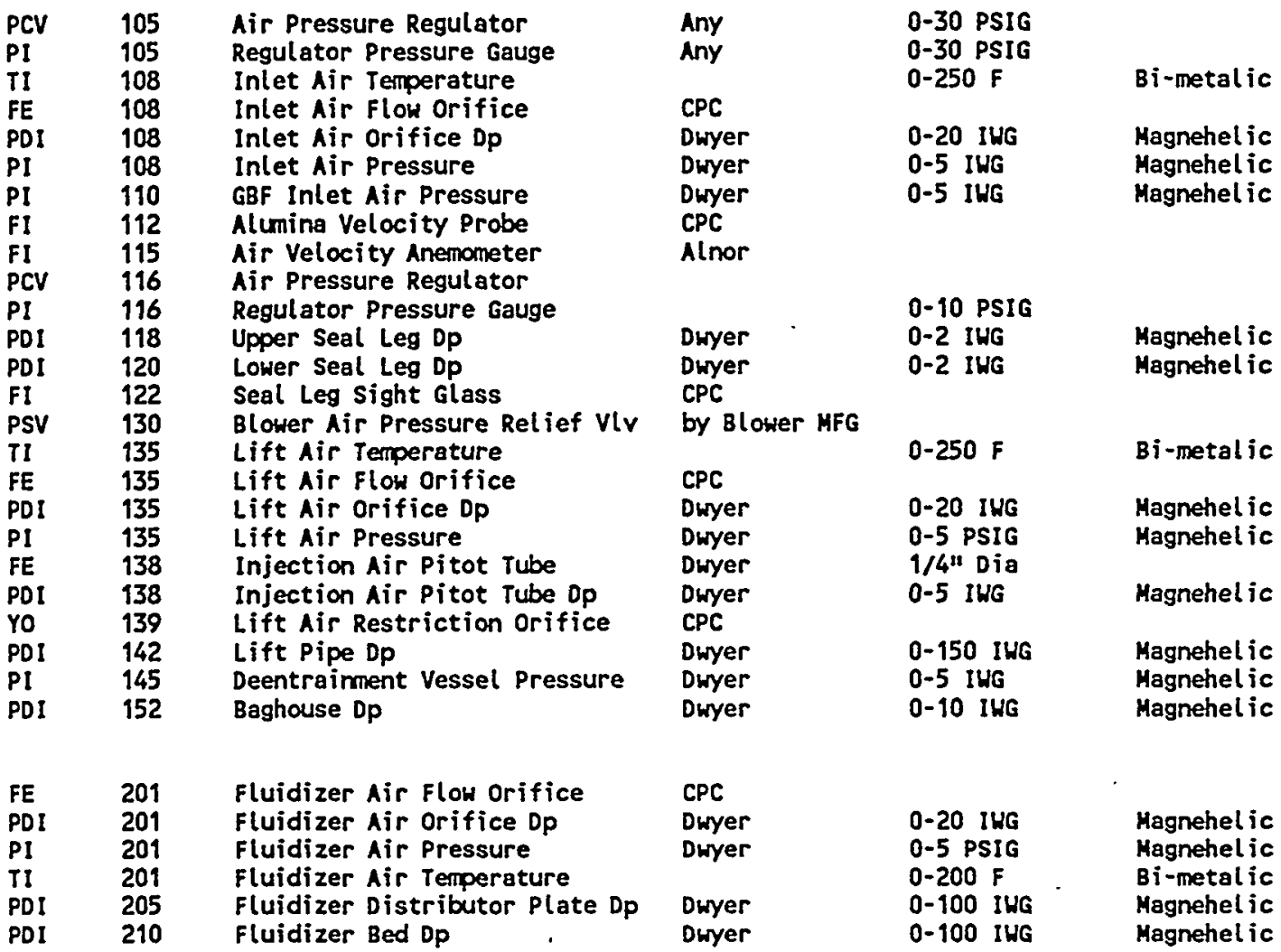

\section{VALVES :}

$\begin{array}{lll}\text { HV } & 101 & \text { Blower Ho.1 Inlet Valve } \\ \text { HV } & 102 & \text { Blower Ho.2 Inlet Valve } \\ \text { HV } & 105 & \text { Ash Eductor Air Valve } \\ \text { HV } & 116 & \text { Bleed Eductor Air Valve } \\ \text { HV } & 117 & \text { Bleed Isolation Valve } \\ \text { HV } & 132 & \text { Lift Air Vent Valve } \\ \text { HV } & 138 & \text { Injection Air Valve } \\ \text { HV } & 155 & \text { Baghouse Hopper Valve } \\ \text { HV } & 158 & \text { Suction Fan Inlet Valve }\end{array}$

Dezurick or Eq. 8" Dia

Dezurick or Eq. 8" Dia

Any

Any

Any

Any

Any

Fabri or Eq.

Dezurick or Eq

2" Dia

3" Dia

8 " Dia
3/4" Dia

3/4" Dia

1 1/2" Dia

Suction Fan Inlet Valve
Butterfly

Butterfly

Ball

Ball

Ball

Gate

Gate

Gate

Butterfly 
6.0 UTILITY REQUIREMENTS

6.1 Compressed Air:

Ash Eductor Air

Bleed Eductor Air

Baghouse Pulse Air

51 SCFM 25 psig

36 SCFM a 5 psig

5 SCFM 80 psig

Total Air

92 SCFM

6.1 Electrical: (Installed Horsepower)

Hoffman Blower Motor Lamson Blower Motor

Suction Fan Motor

Lift Air Blower Motor

Ash Feeder Motor

Drum Rotator
125 HP a 230VAC, 3 phase $100 \mathrm{HP}$ 230VAC, 3 phase 5 HP a 23OVAC, 3 phase 15 HP 230VAC, 3 phase $1 / 2$ HP @ 115VAC, 1 phase $1 / 2$ HP \& 115VAC, 1 phase

6.2 Diesel Fuel:
Air Compressor
$1.5-2 \mathrm{Gal} / \mathrm{Hr}$ 


\subsection{ESTIMATED COSTS}

7.1 Task 5: Fabrication and Installation Costs:

Fabrication and installed costs are defined below using the cost format from the option I cost Proposal submitted to the U.S. Department of Energy during April, 1994. Some of the equipment costs are higher than originally estimated based on fabricator quotations. Also, some additional equipment must be rented that were originally thought to be available at CPC. These additional costs are listed separately. Every effort will be made to reduce costs in other areas to compensate for cost increases. Section 7.2 provides a detailed breakdown of the cost increases.

\section{April-1994 \\ Cost Proposal Additional. Estimate \\ Costs}

Item 1a: Purchased Parts

Item 1b: Subcont. Items

Item 3: Direct Labor

Item 4: Labor Overhead

Item 7: Travel

$\$ 37,600$
$\$ 130,035$
$\$ 10,172$
$\$ 15,258$
$\$ 44$

$\$ 37,600$

$\$ 130,035$

$\$ 15,258$

$\$ 44$
$\$ 11,240$

$\$ 6,460$

$\$ 0$

$\$ 0$

$\$ 0$

April 1994 Total Task 5 Direct Cost and Overhead \$193,109

Revised Task 5 Total Direct Cost and overhead $\$ 210,809$

7.2 Task 5 Detail of Cost Changes

Item 1a: Purchased Parts

Addition: Added Costs for Half Filter Assy. $\$ 7,255$

Added Costs for Media Fluidizer $\$ 1,235$

Added Costs for Liner Abrasion

Unit (due to sampling preparation) $\$ 2,750$

$$
\text { Total } \$ 11,240
$$

Item 1b: Subcontracts

$$
\begin{array}{llll}
\text { Addition: } & \text { Rental of } \mathrm{PD} B \mathrm{~B} \text { ower } 9 \$ 400 / \mathrm{mo} & \$ 1,600 \text { (4 mo) } \\
\text { Rental of Ash Feeder } \$ \$ 465 / \mathrm{mo} & \$ 1,860 \text { (4 mo) } \\
\text { Rental of Air Compressor e } \$ 1000 / \mathrm{mo} \$ 3,000 \text { (3 mo) }
\end{array}
$$

7.3 Total OPTION I costs

The current cost trend for Option 3 is upward by about 5\% based on the above changes in above costs. This is only a small part of the total contract. There are lots of opportunities to offset this trend in other tasks and stay 
within budget. 


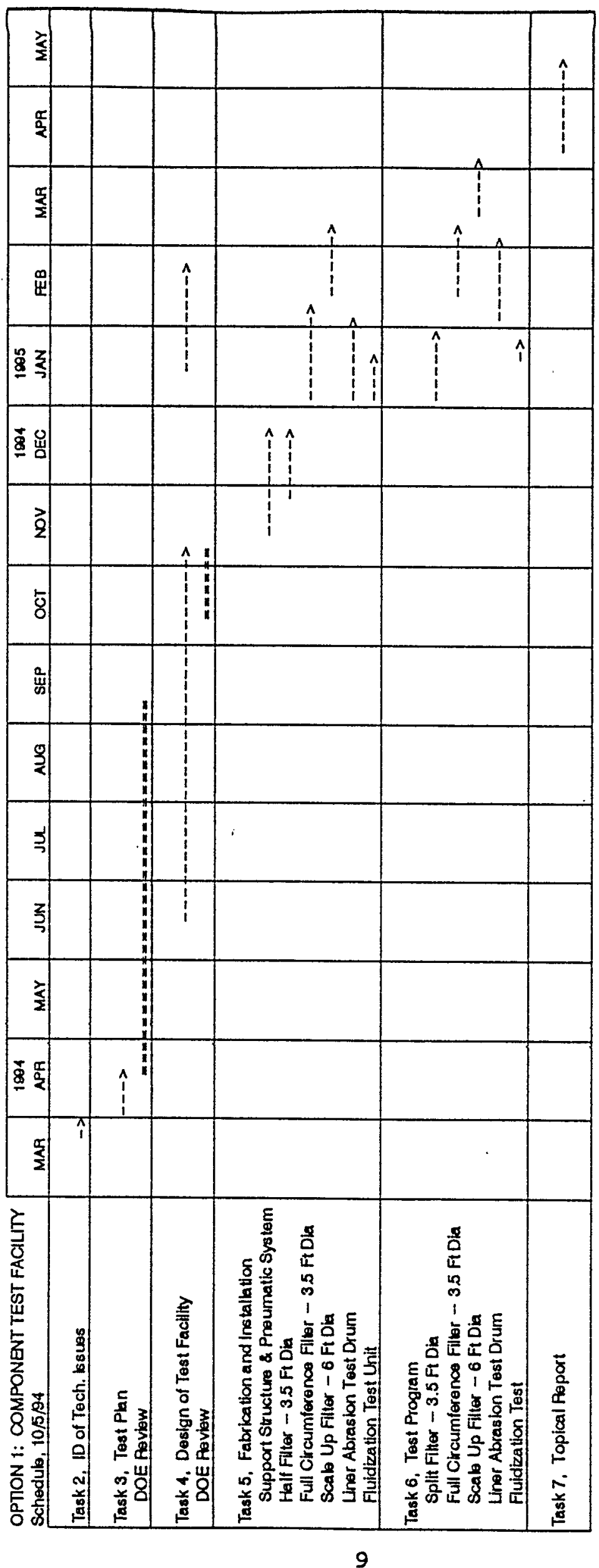




\subsection{ENGINEERING DRAWINGS}



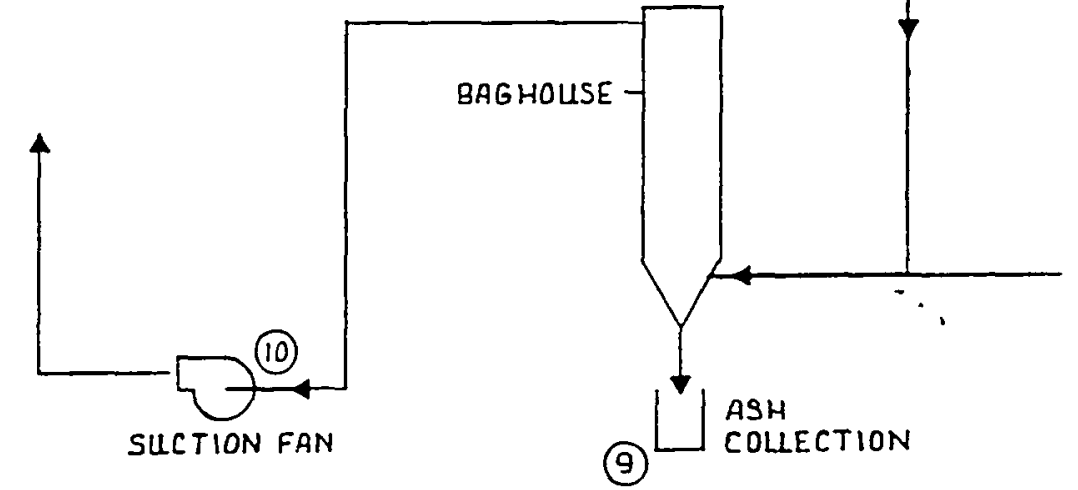

(8)

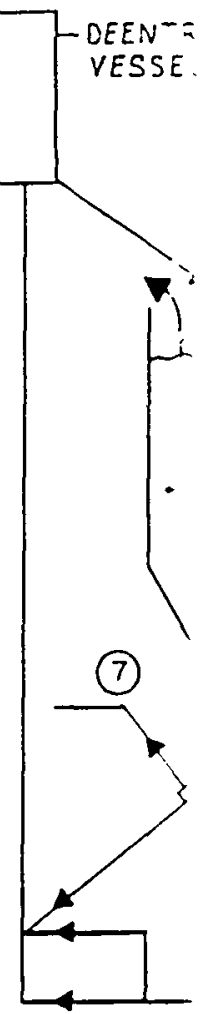

ESTIMATED MAXIMUM FLOW CONDITIONS

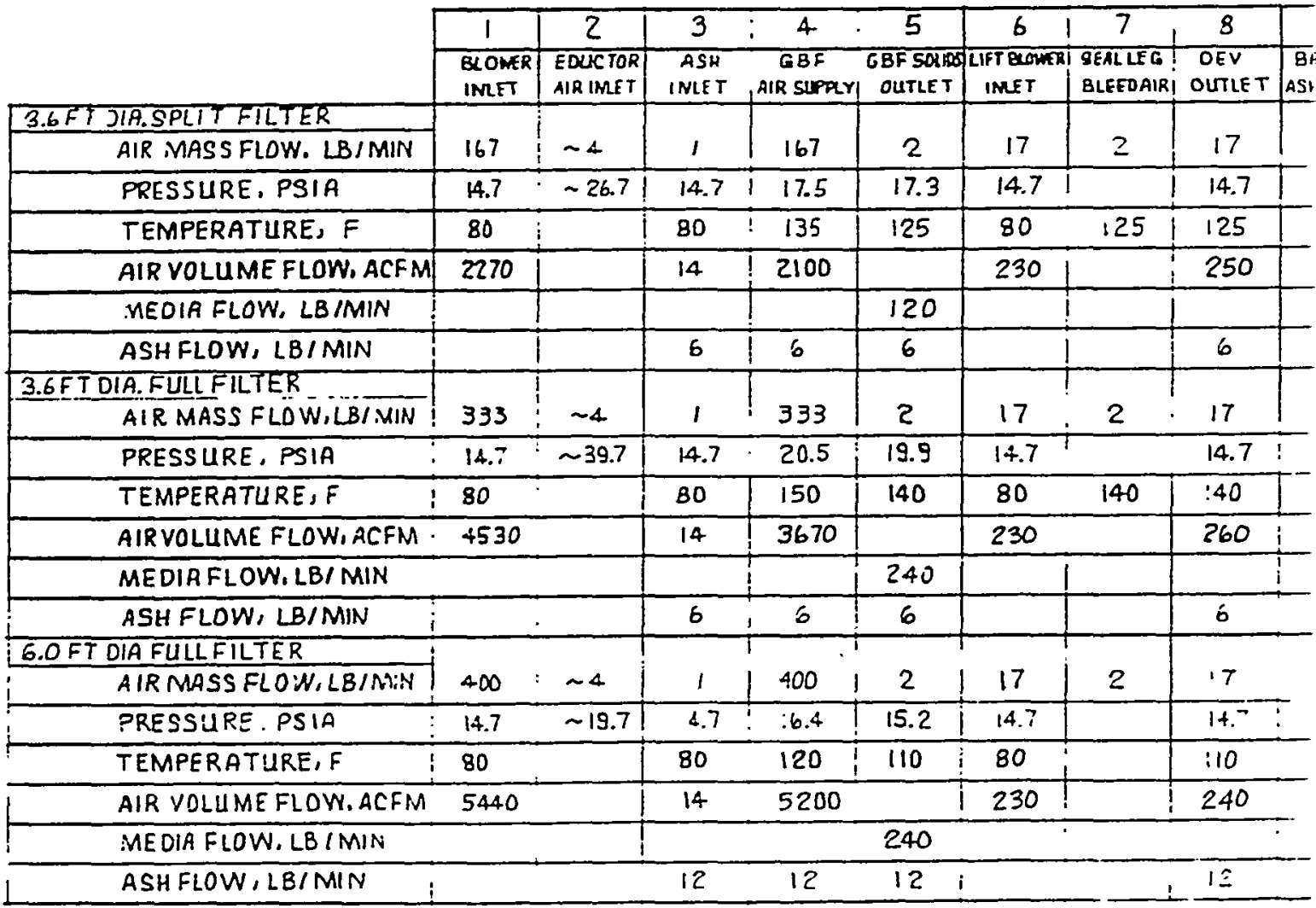




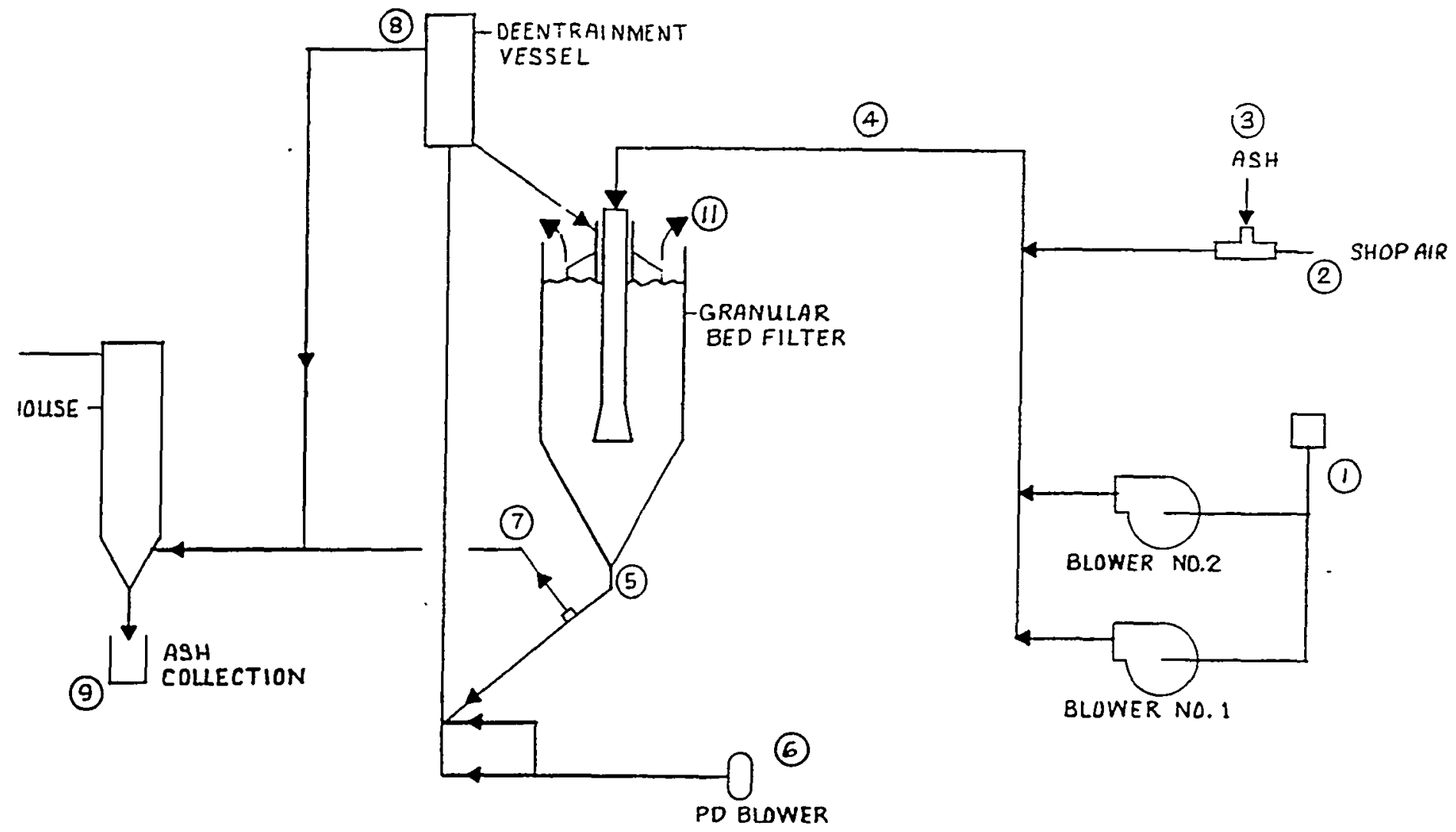

TED MAXIMUM FLOW CONDITIONS

\begin{tabular}{|c|c|c|c|c|c|c|c|c|}
\hline & $\vdots 4$ & 5 & 6 & 7 & 8 & 9 & 10 & \multirow{2}{*}{\begin{tabular}{|c|}
11 \\
GBF \\
OUTLET
\end{tabular}} \\
\hline ET & $\begin{array}{l}\text { GBF } \\
\text { AIR SUPAYY }\end{array}$ & $\begin{array}{l}\text { CBF SODOS } \\
\text { OUTLET }\end{array}$ & $\begin{array}{l}\text { LIFT Deowin } \\
\text { IMET }\end{array}$ & $\begin{array}{l}\text { SEALLFG } \\
\text { BLFEOAIRI }\end{array}$ & $\begin{array}{l}\text { DEV } \\
\text { OUTLET }\end{array}$ & \multicolumn{2}{|c|}{$\begin{array}{l}\text { BAGHOUSET SUCTION } \\
\text { ASH PEMOMUIFAN INET }\end{array}$} & \\
\hline & 167 & 2 & 17 & 2 & 17 & & 19 & 167 \\
\hline .7 & 117.5 & 17.3 & 14.7 & & 14.7 & & 14.1 & 14.7 \\
\hline ' & $! \quad 135$ & 125 & 80 & 125 & 125 & & i 120 & 70 \\
\hline & 2100 & & 230 & & 250 & & 290 & 2230 \\
\hline & & 120 & & & & & i & \\
\hline & 6 & 6 & & & 6 & 6 & $<.03$ & $<.06$ \\
\hline & 333 & 2 & 17 & 2 & 17 & & 19 & : 333 \\
\hline .7 & 20.5 & 19.9 & 14.7 & & 14.7 & $!$ & 14.0 & 14.7 \\
\hline 1 & 150 & 140 & 80 & 140 & $: 40$ & & 135 & 170 \\
\hline - & 3670 & & 230 & & 260 & 1 & 300 & 14440 \\
\hline & & 240 & & & & I & & 1 \\
\hline & 6 & 6 & & & 6 & 6 & $<.03$ & $:<.06$ \\
\hline & 400 & 2 & 17 & 2 & 17 & : & 19 & 400 \\
\hline 7 & $: 6.4$ & 15.2 & 14.7 & & 14.7 & 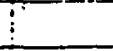 & 14.2 & 14.7 \\
\hline ' & 120 & 110 & 80 & & $: 10$ & $\therefore$ & 110 & 70 \\
\hline . & $5200 !$ & & 230 & & 240 & & 280 & 5330 \\
\hline & & 240 & & & . & . & $i$ & \\
\hline & 12 & 12 & & & 12 & 12 & $<.06$ & $<.12$ \\
\hline
\end{tabular}

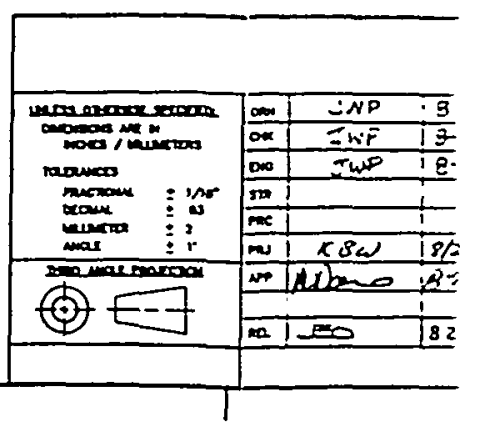


1

(4)

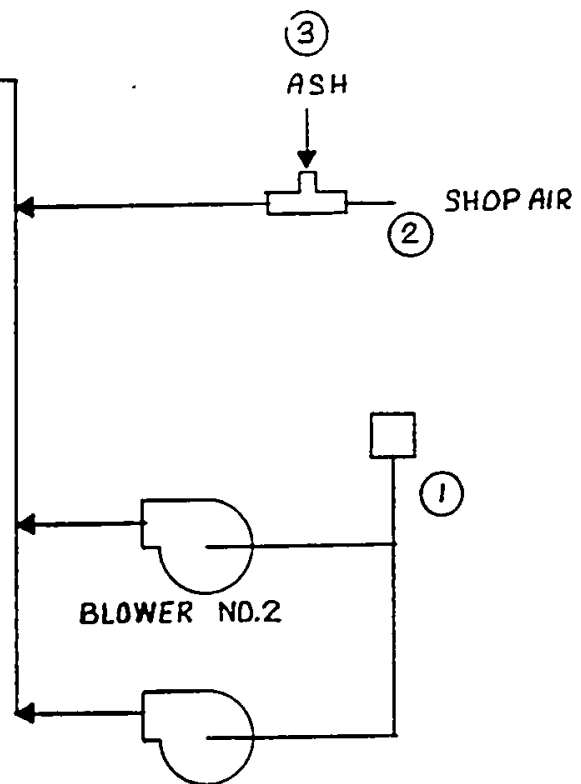

BLOWER NO. 1

DEPARTMENT OF ENERGY

MOVING BED GRANULAR SED FILTER

DEYELDPMENT OROGRAMI

PROCESS FLOW DIAGRAM

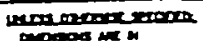

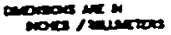

racuess

necrouk ins

nown

$\sin$

0
$\vdots$
$\vdots$
$\vdots$
$\vdots$

(a)

(6) $\subseteq-7$

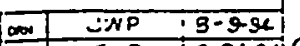

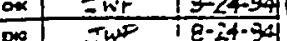

ing

on Tw $18-24-34$

GRANULAR BED FILTER - TEST UNITS

in $x 80$ | $18 / 24 / 94$

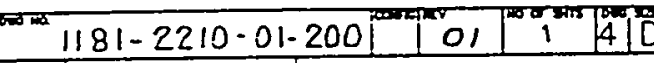

$\rightarrow$ cos $18 / 24 / 94$ |

COMBUSTIIIIN

\begin{tabular}{|l|l|l|}
\hline & EOS & $8.25 \cdot 44$ \\
\hline
\end{tabular}

combuation Poorer Compony. Oationd. Colttomis 


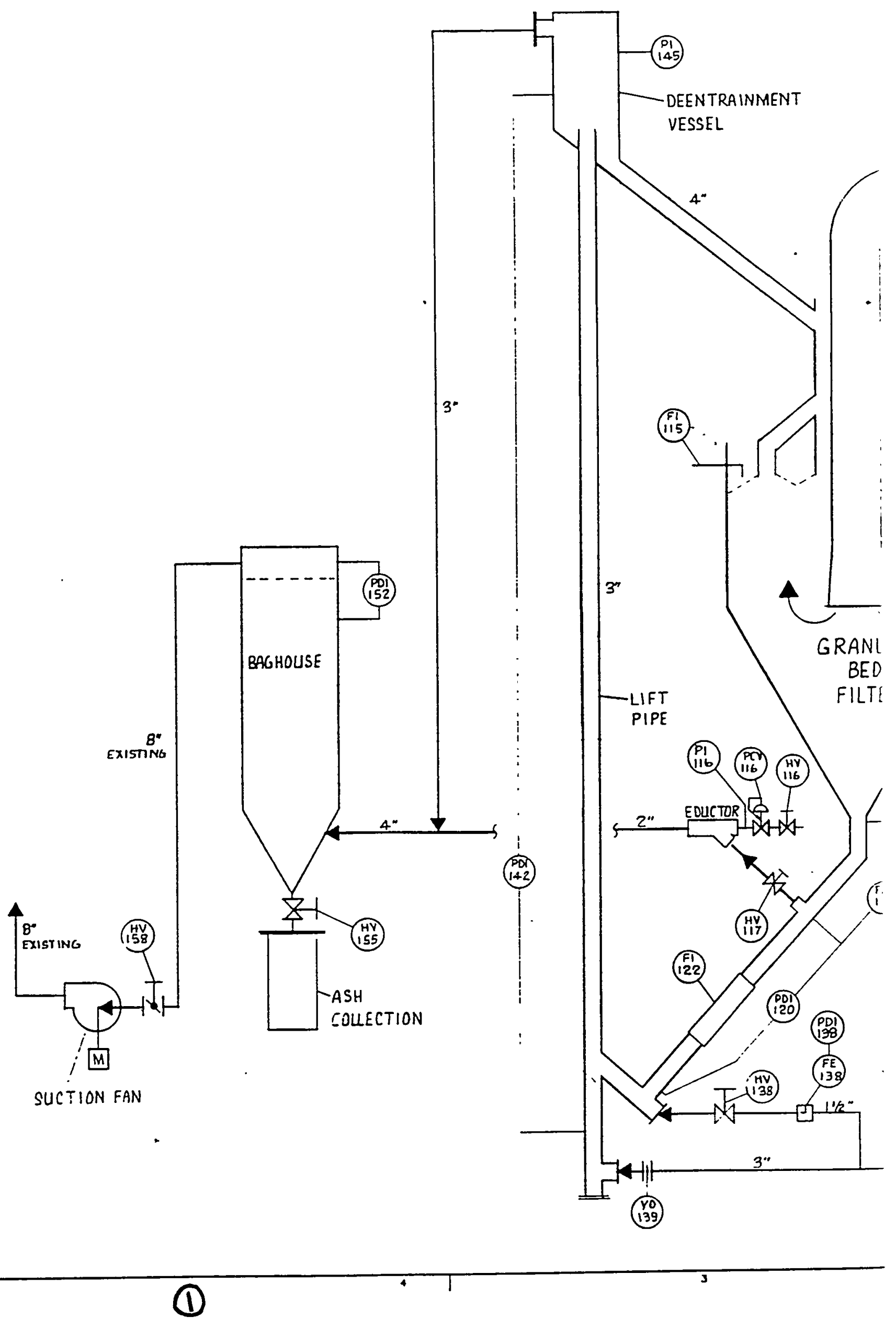




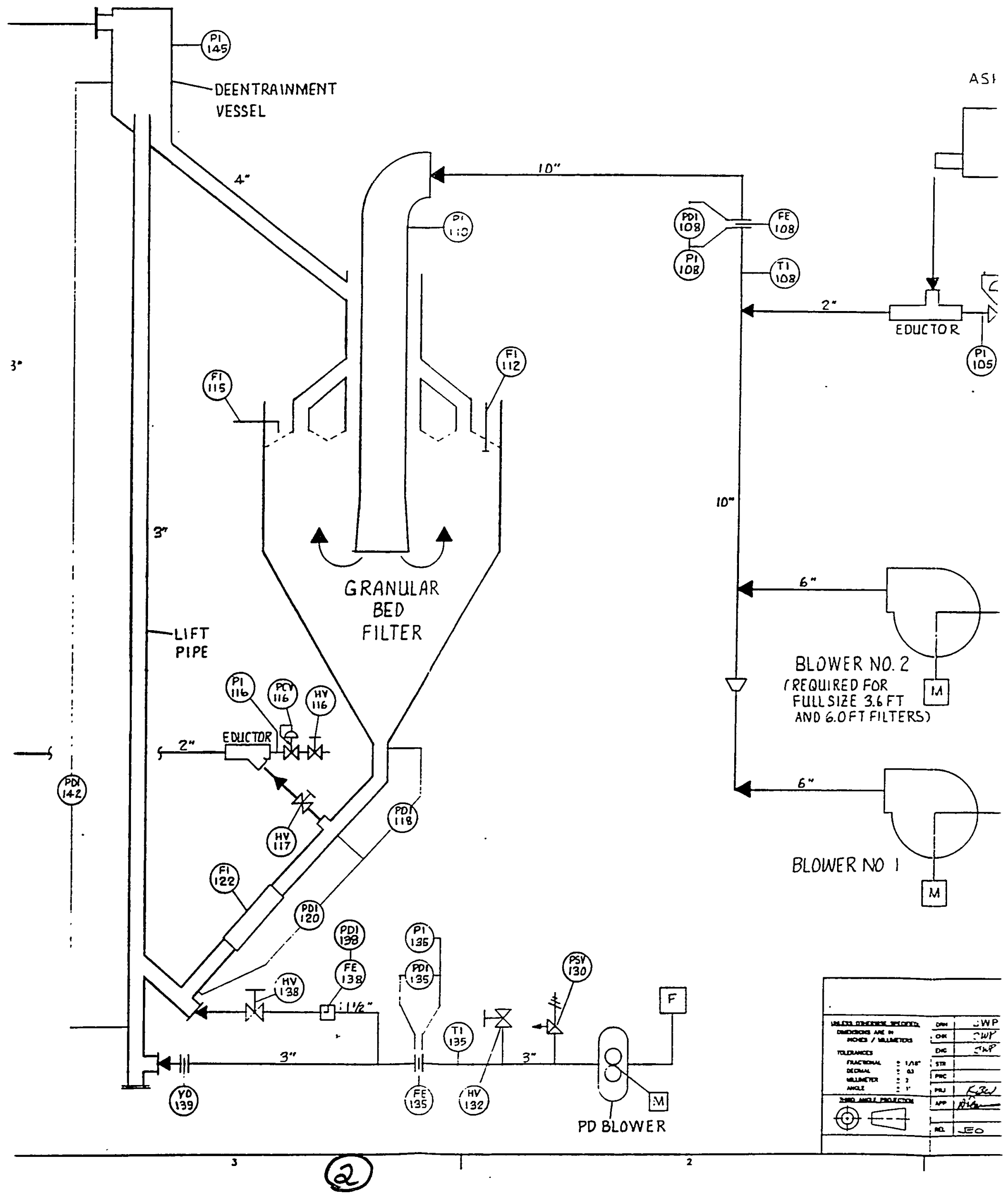




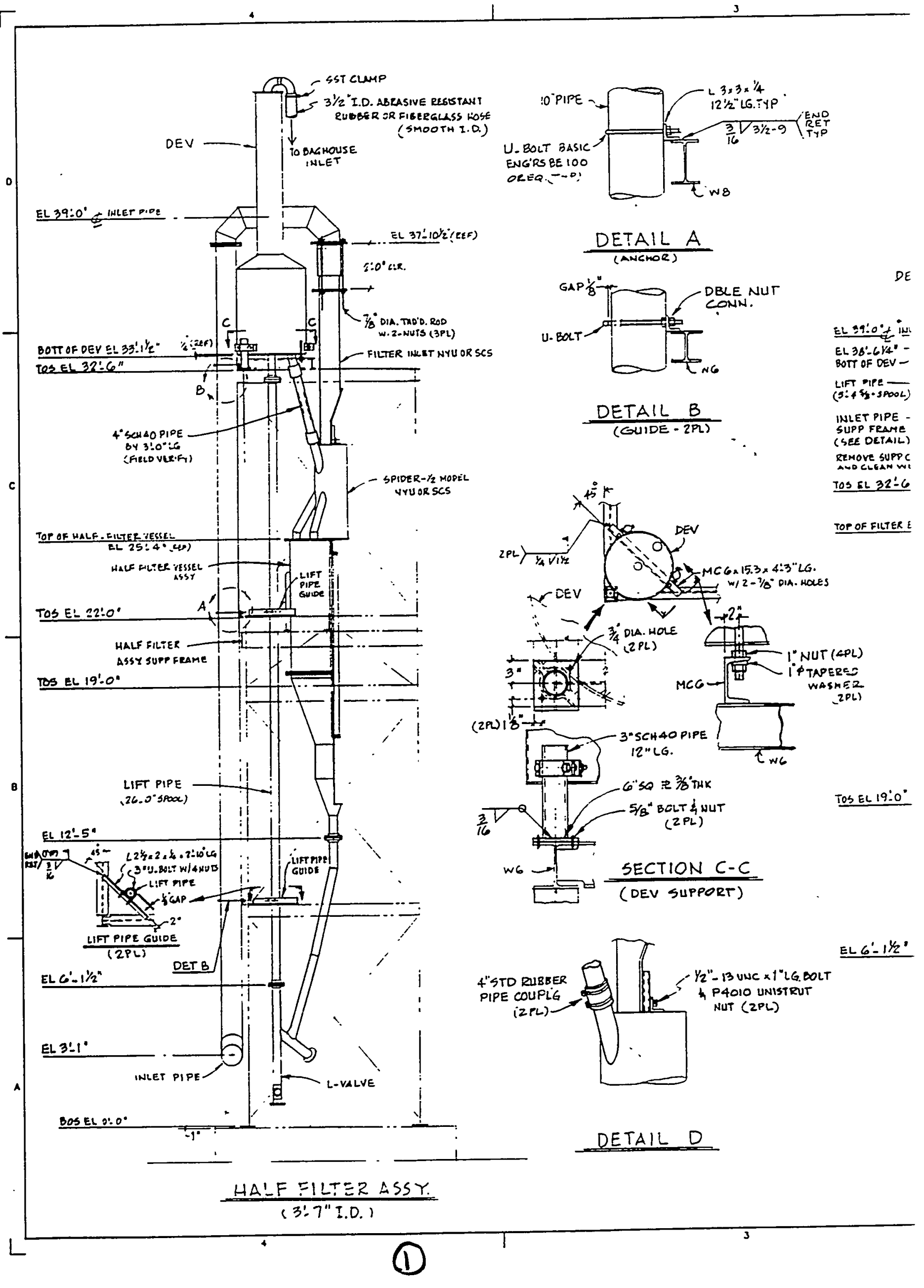



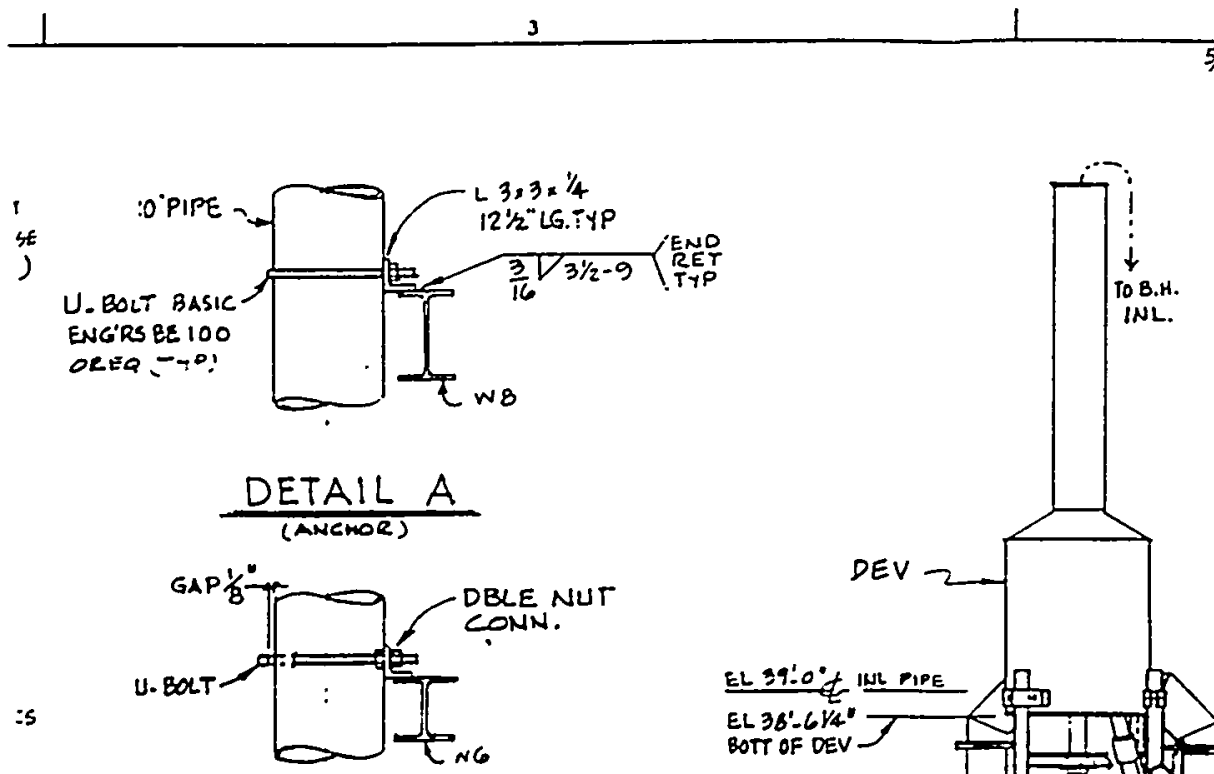

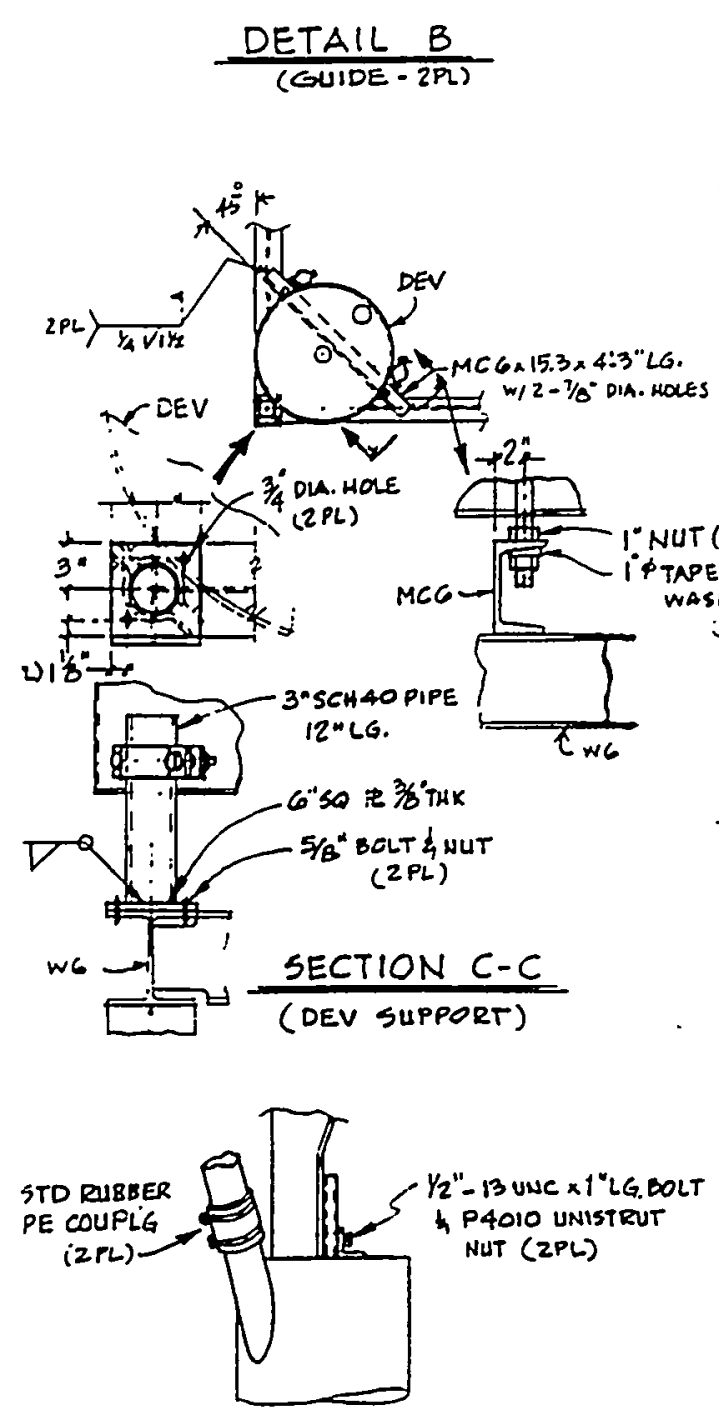

DETAIL D

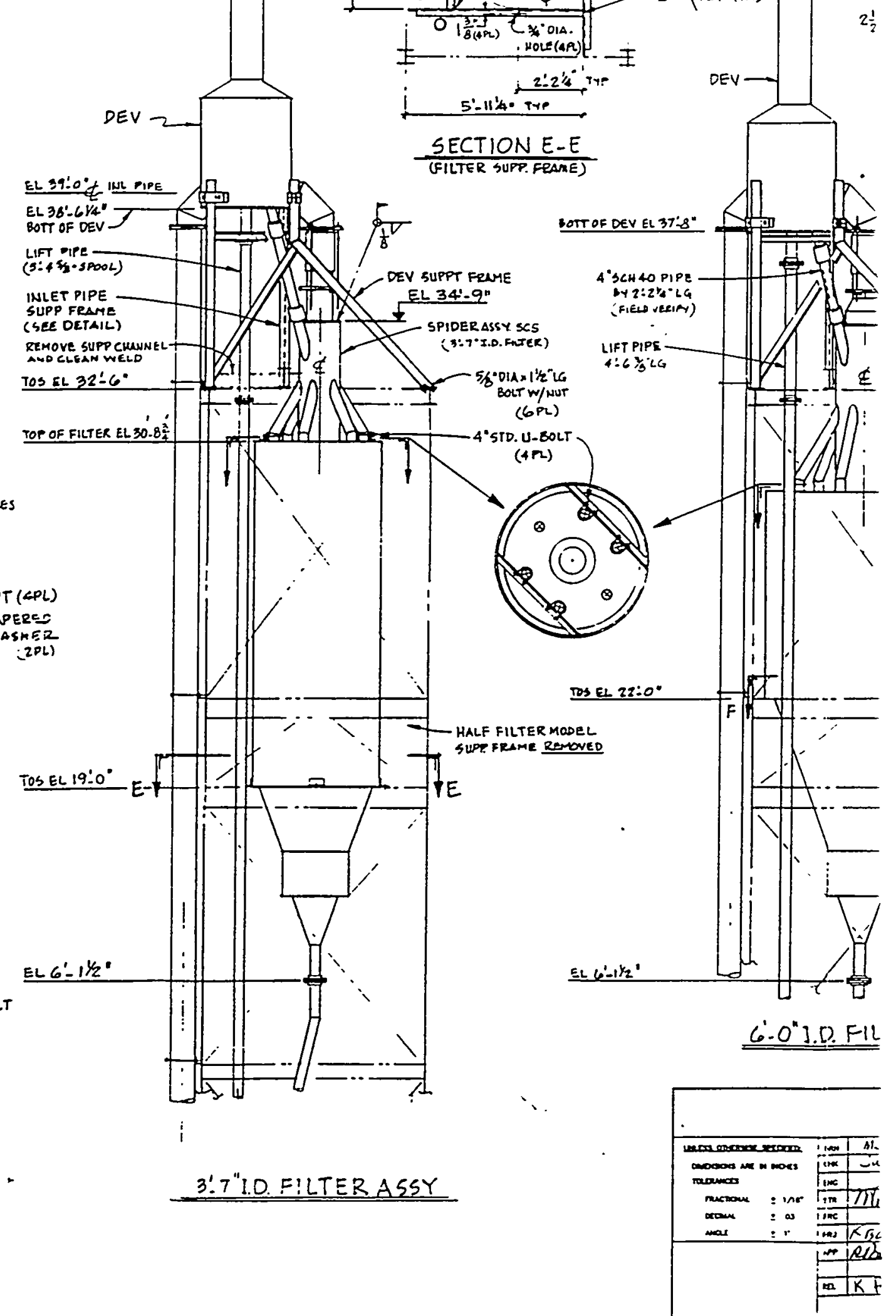


IIU

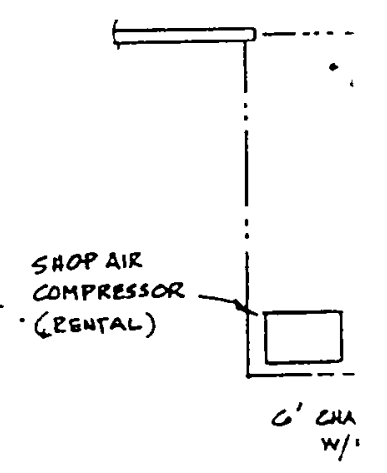

-

1

1

]

1

1

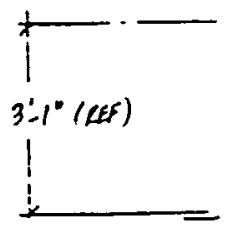




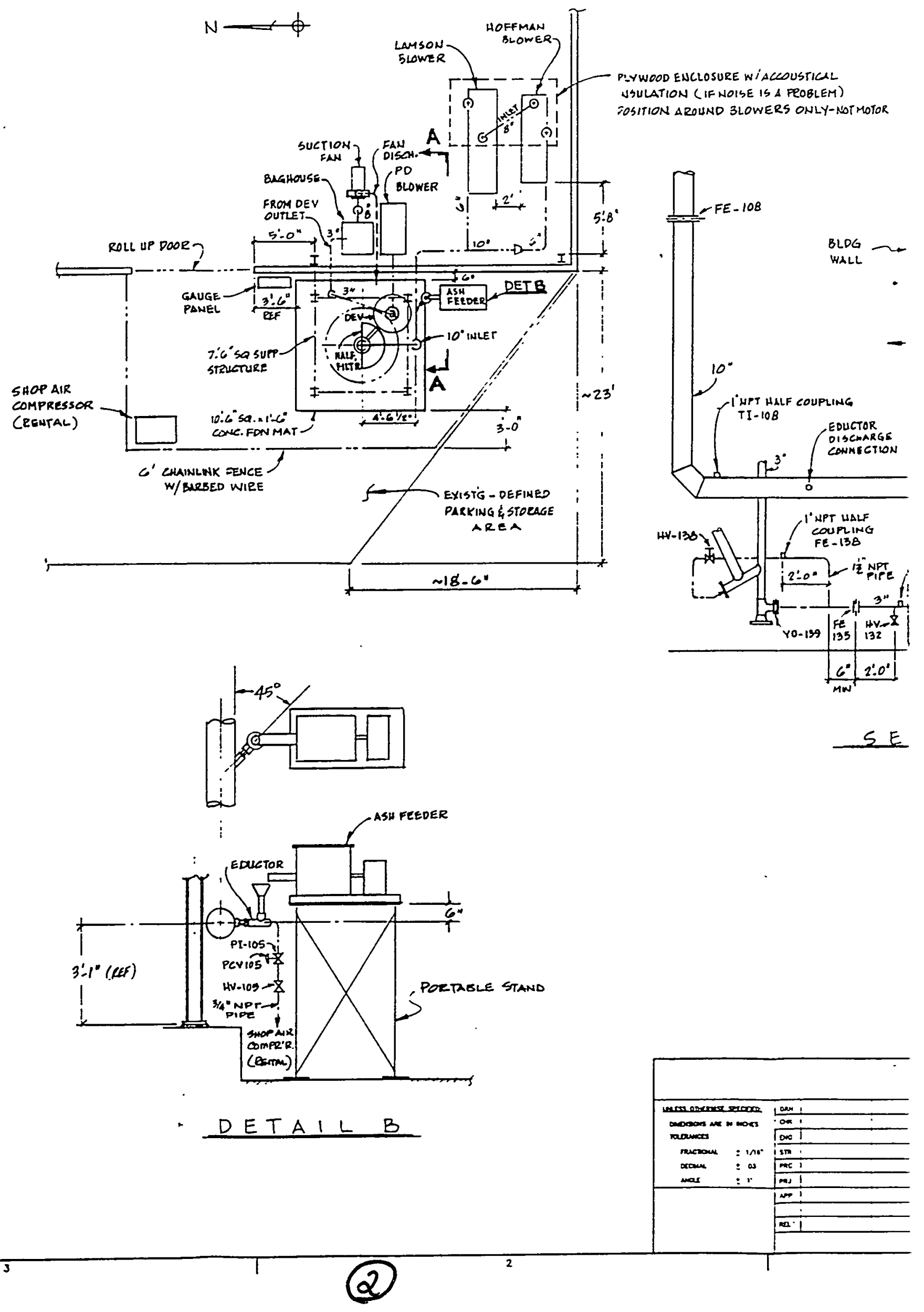




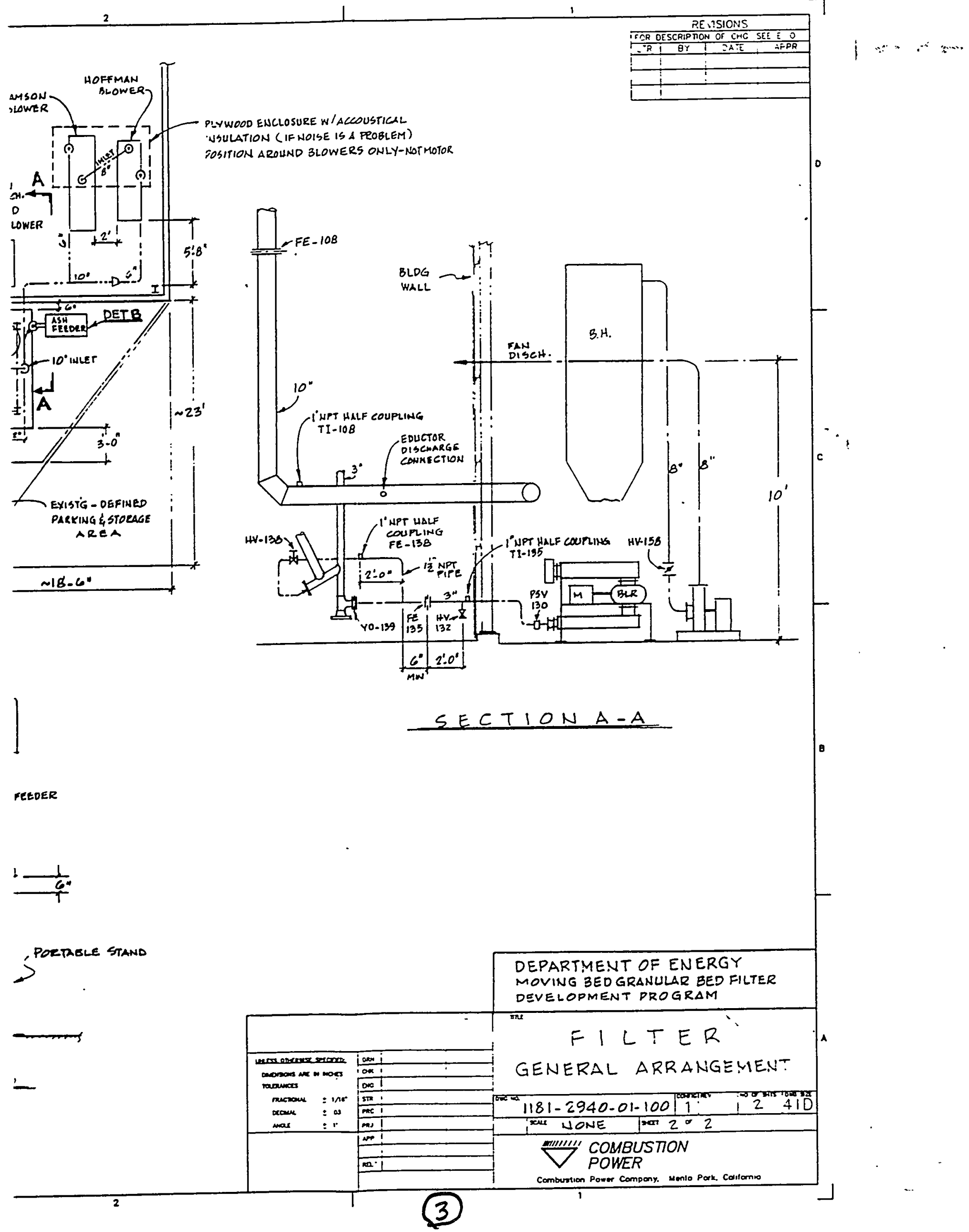




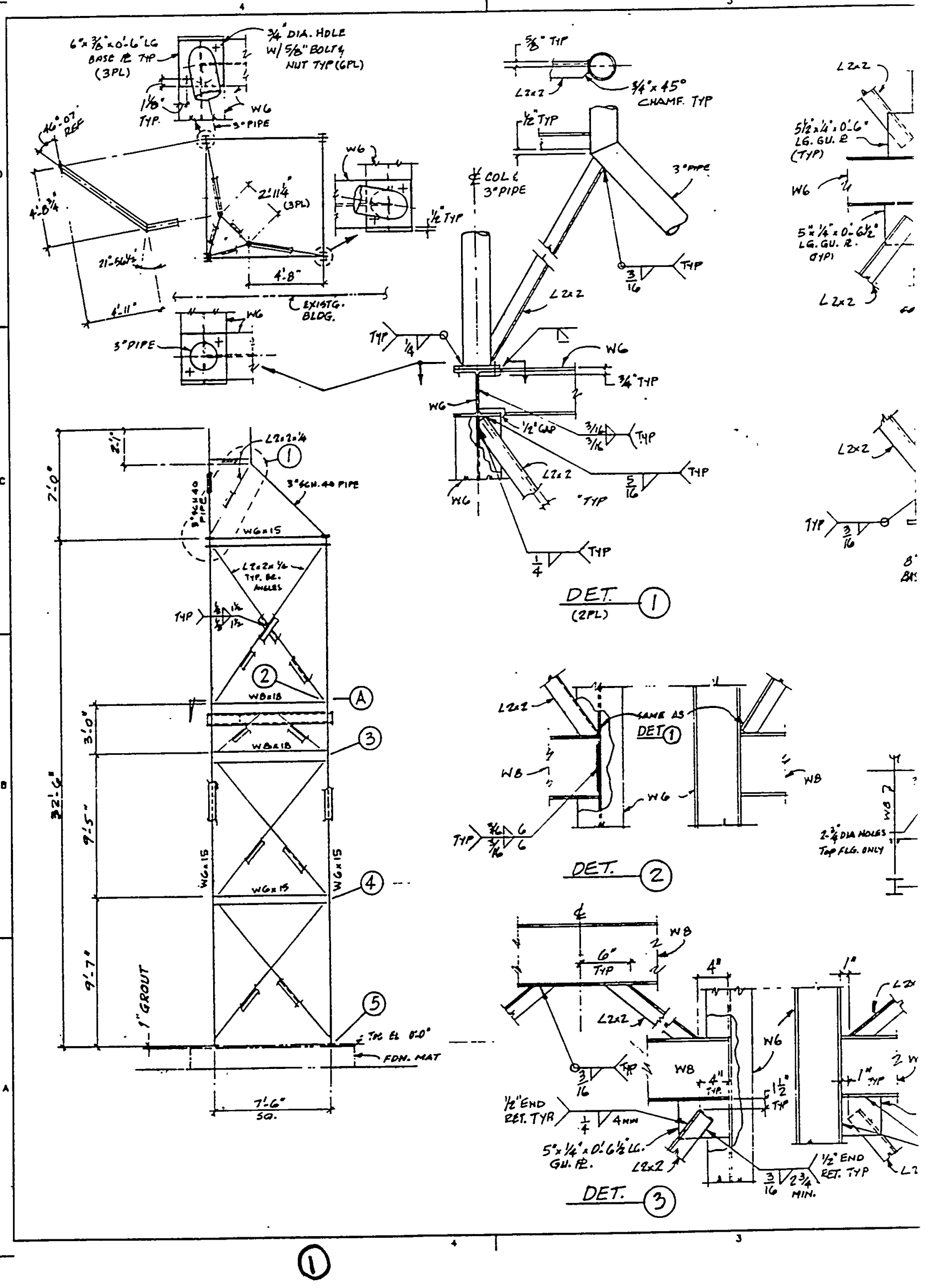



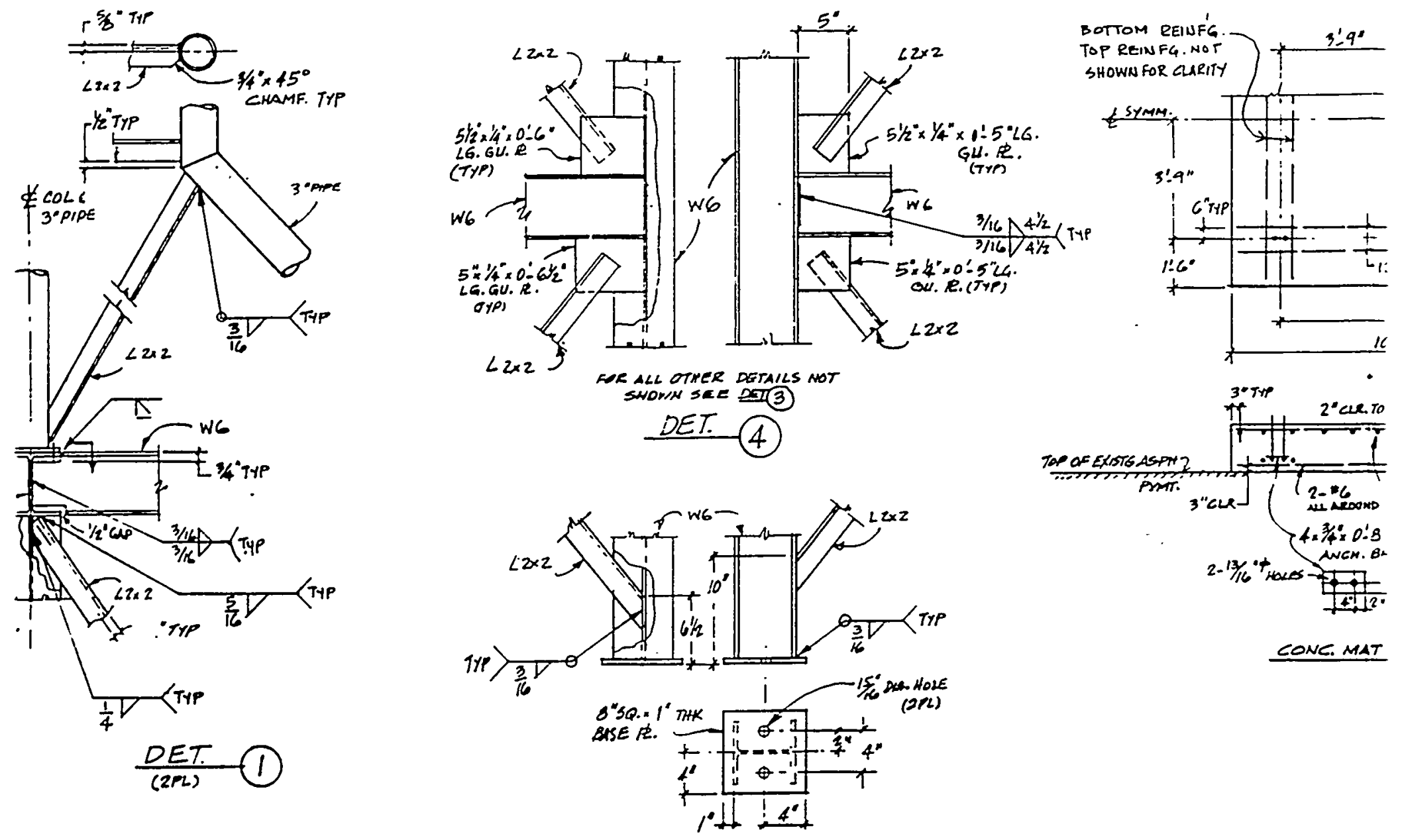

CONC. MAT
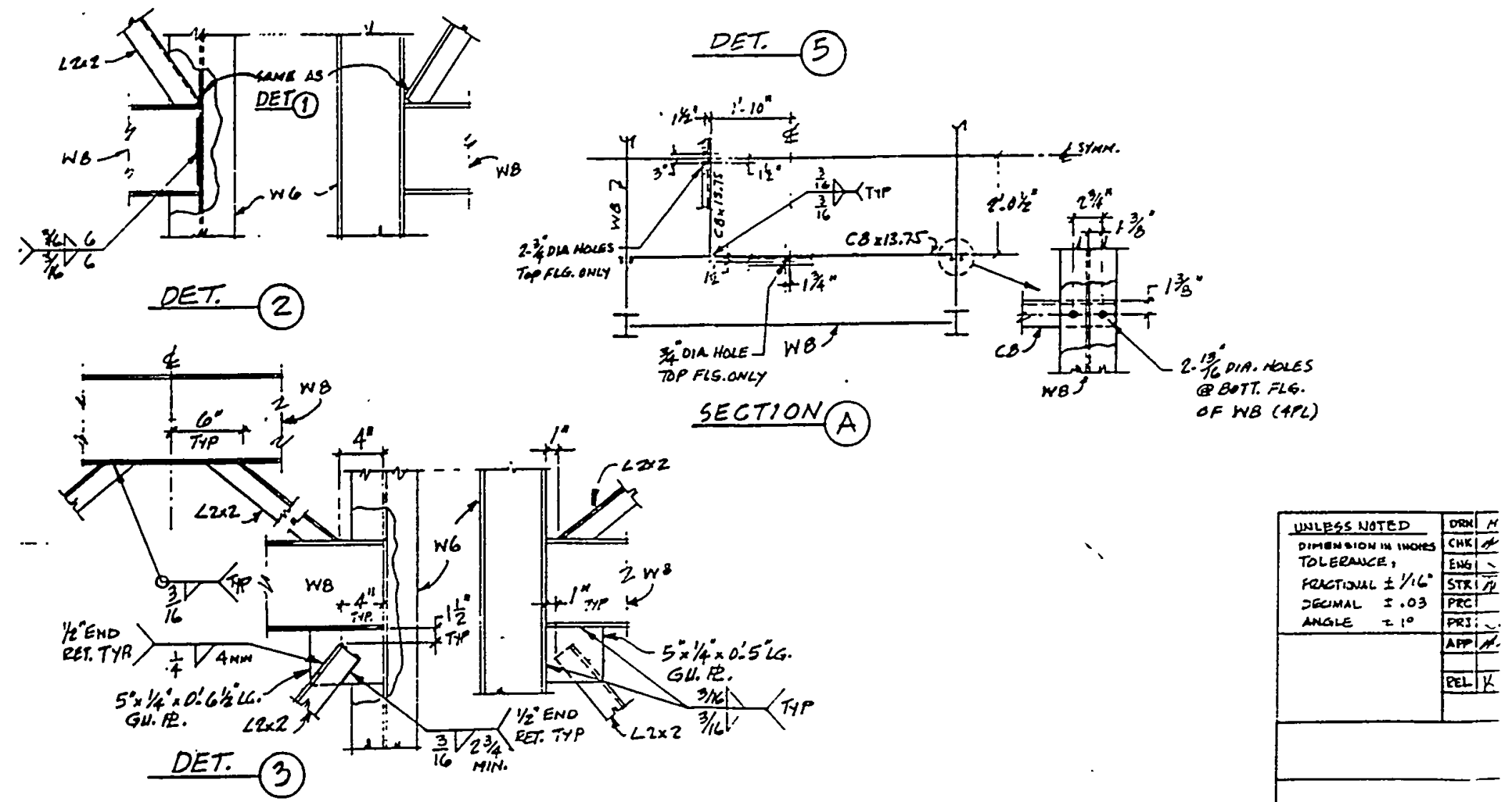


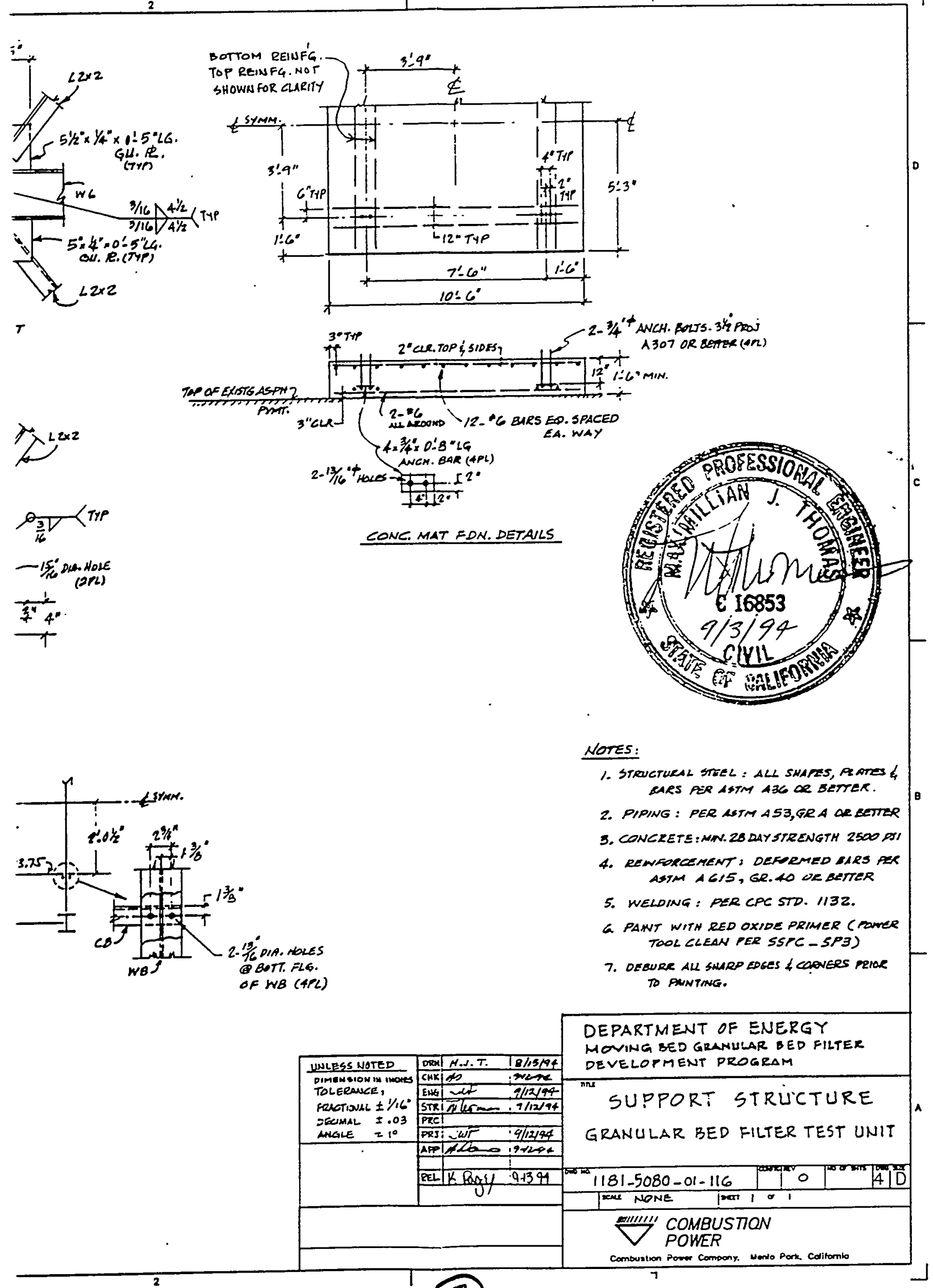




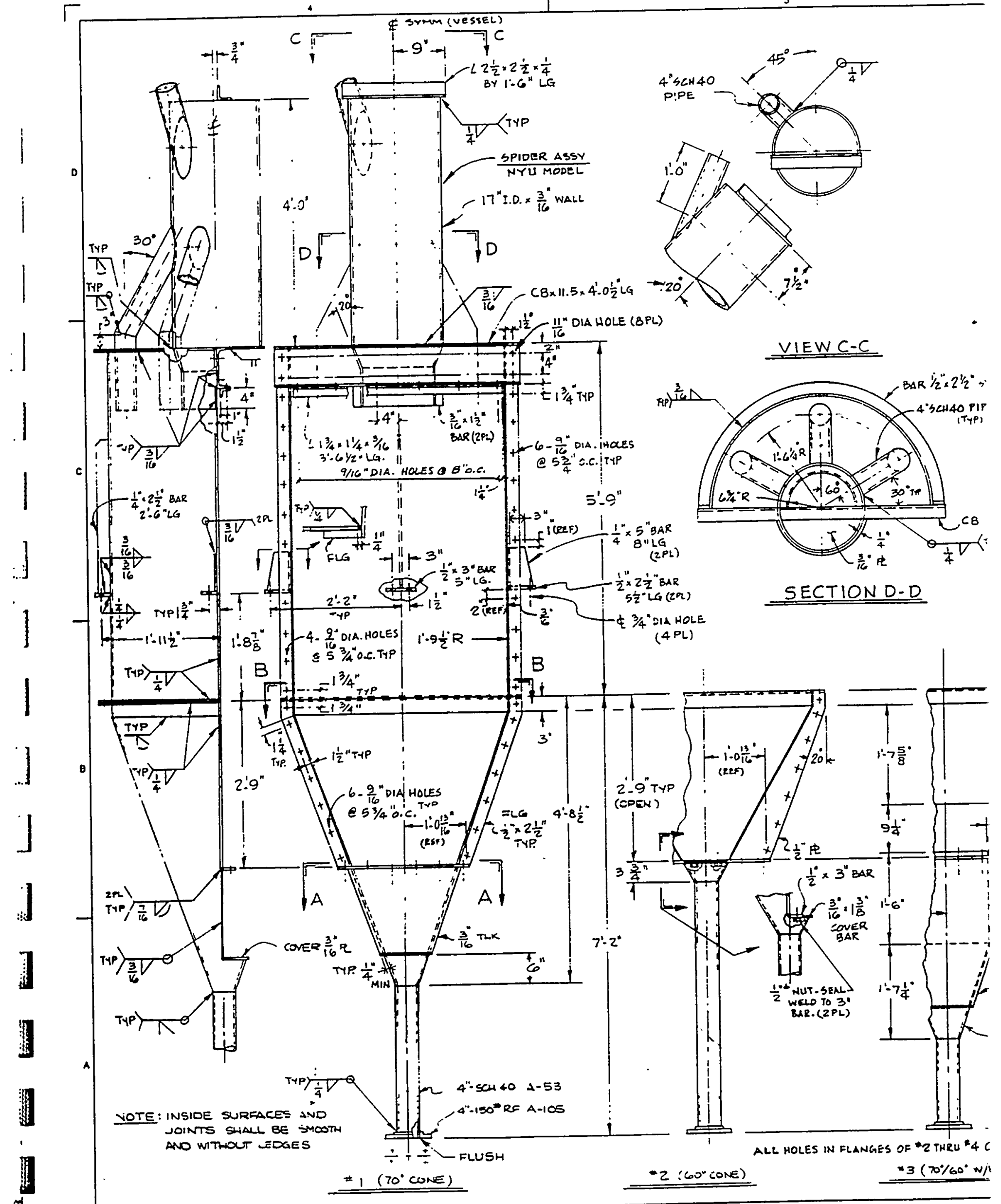




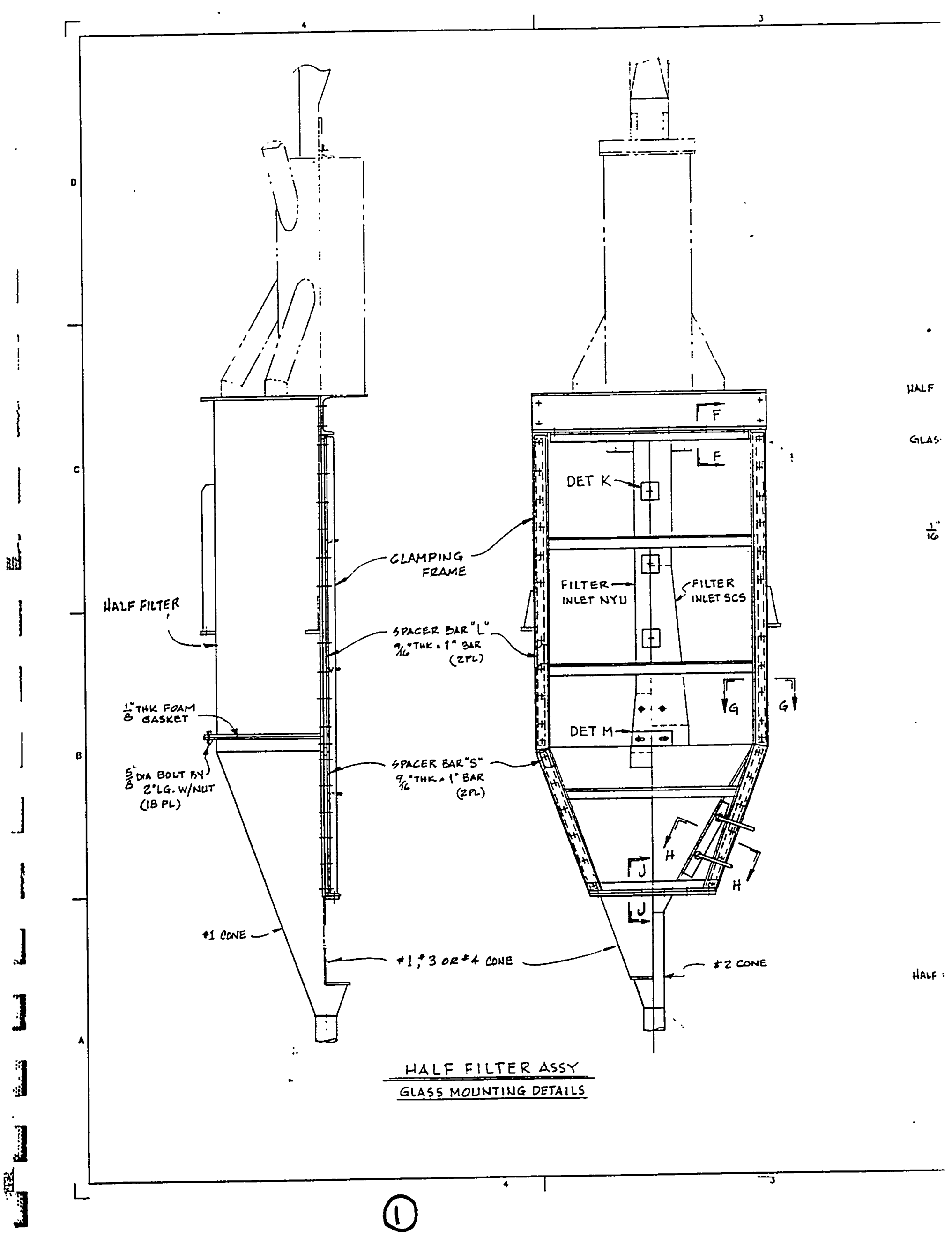




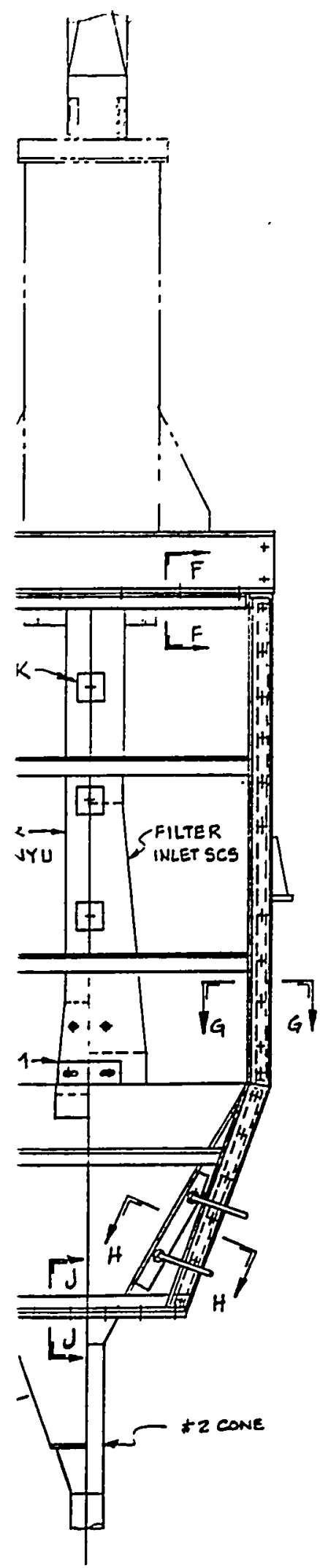

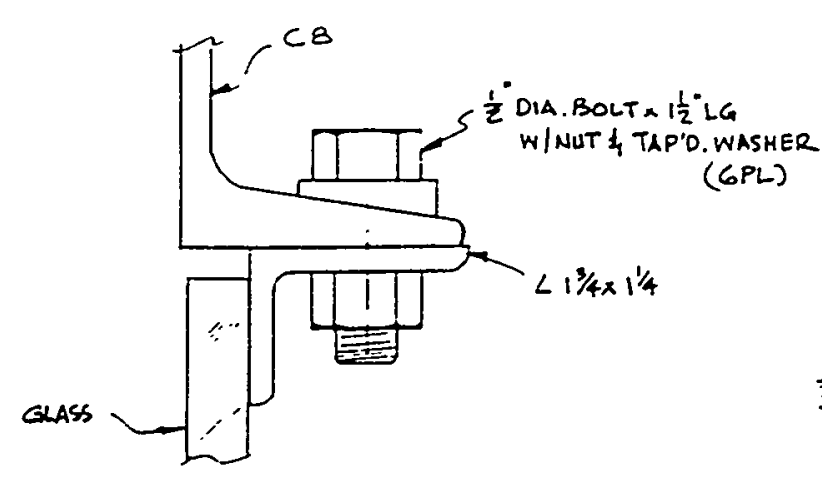

SECTION F-F
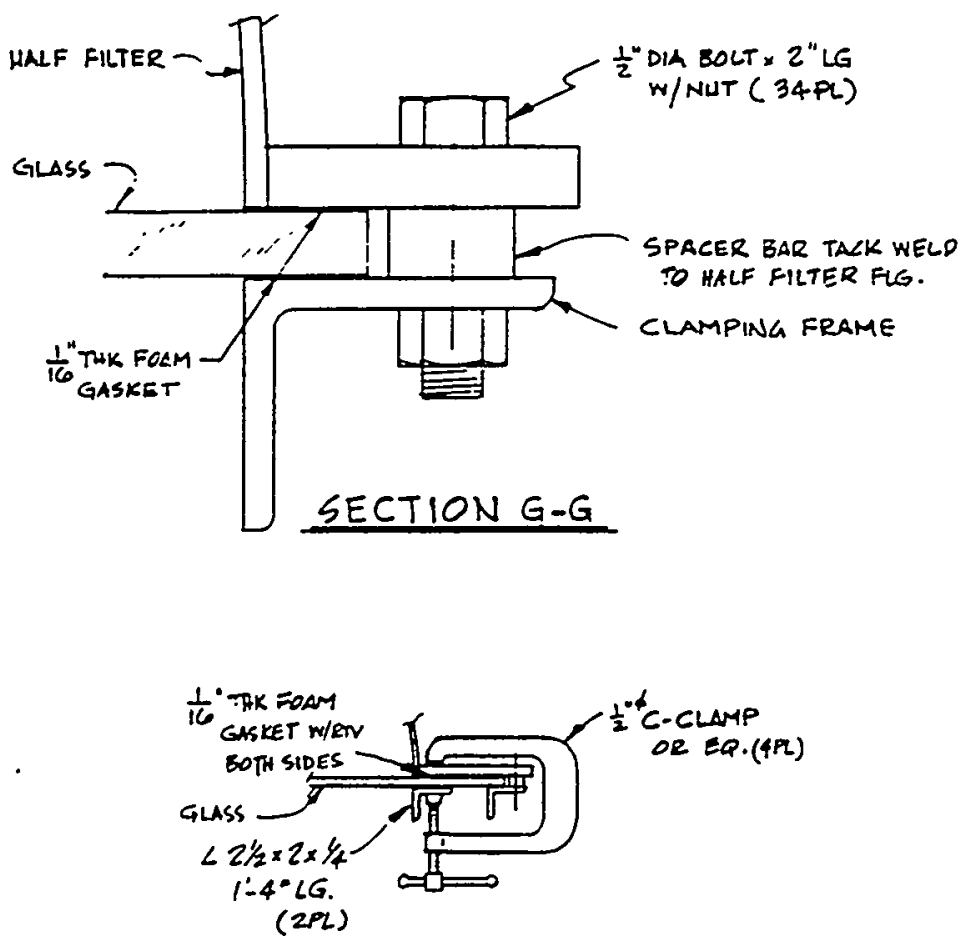

SECTION H-H

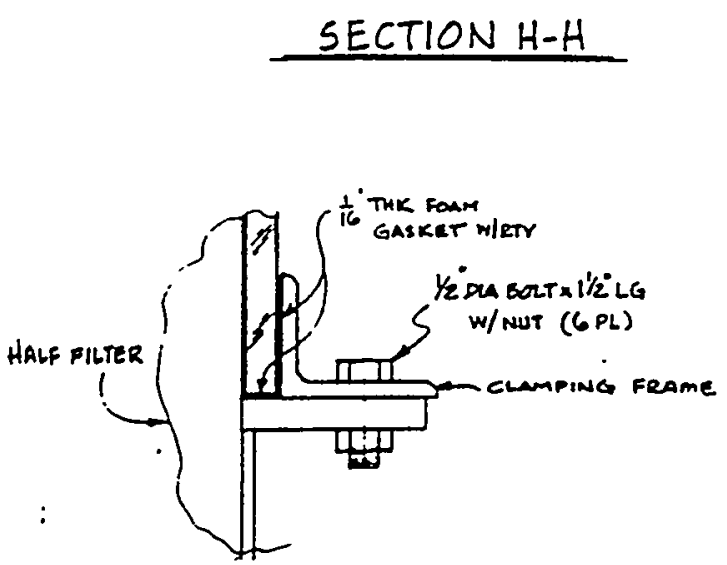

SECTION J-J
FILTER INLET
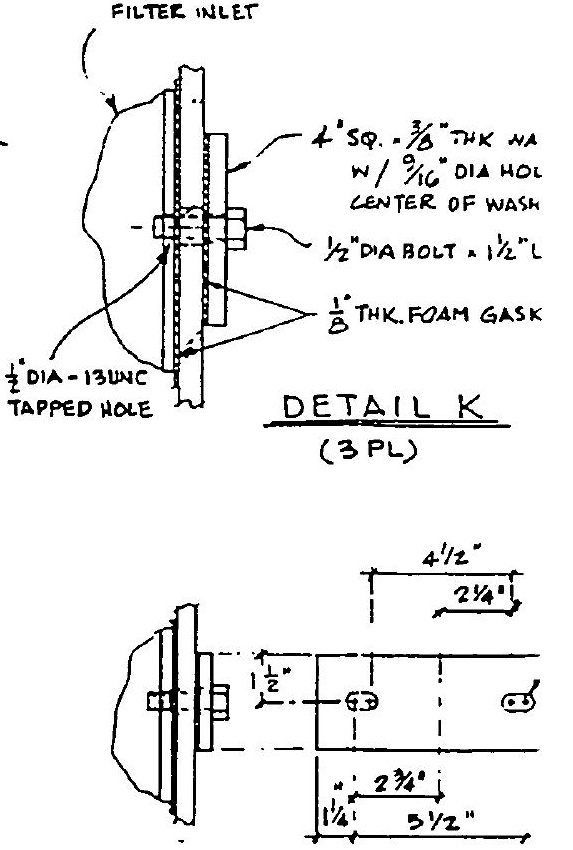

CALL DETAILS NOT SHOWN SA

DETAILM

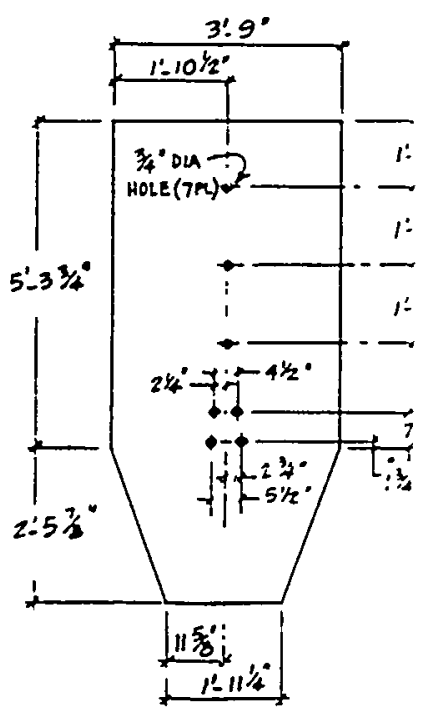

DETAIL OF

$\frac{1}{2}$ "TWX TEMPERED GLASS 


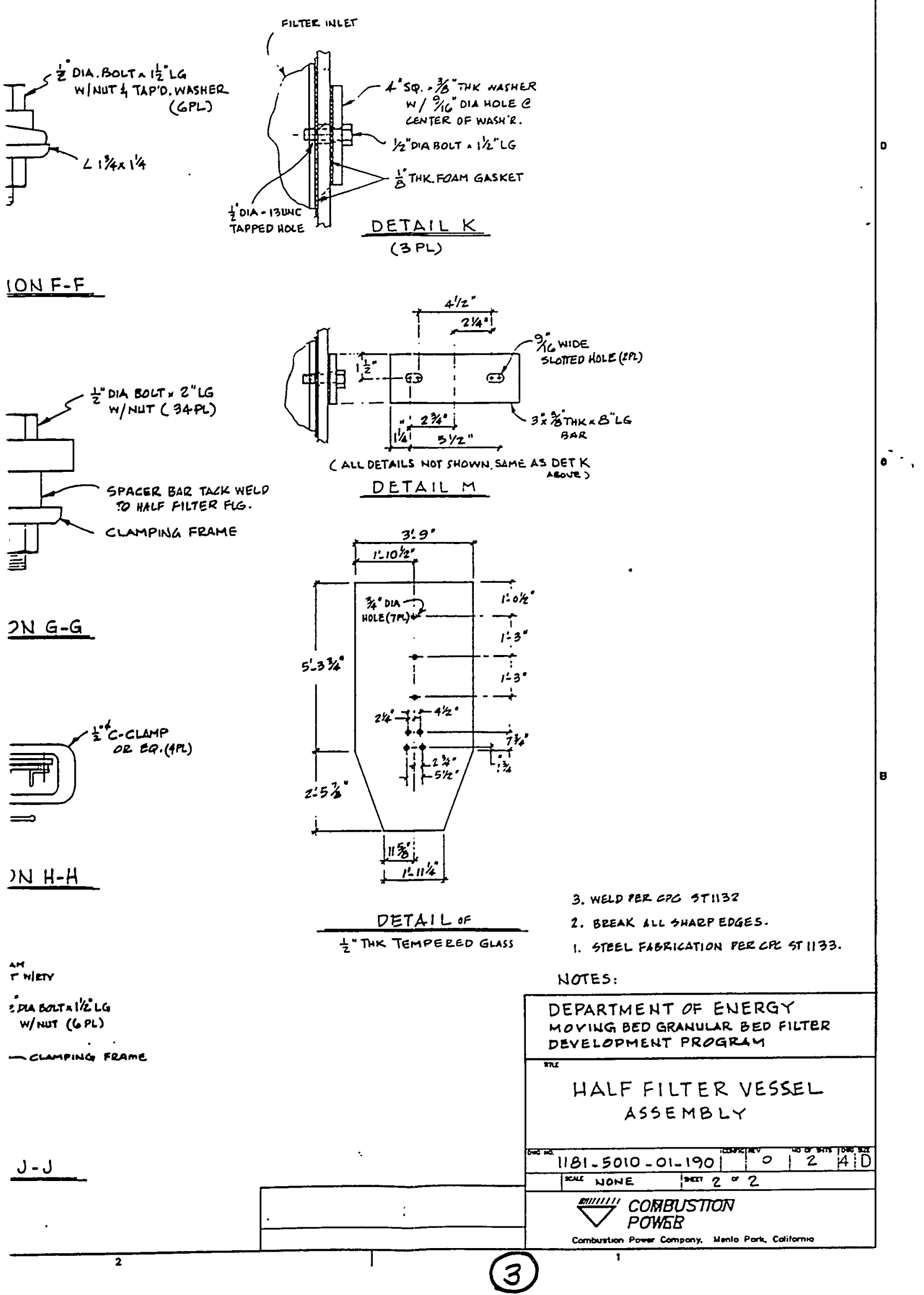




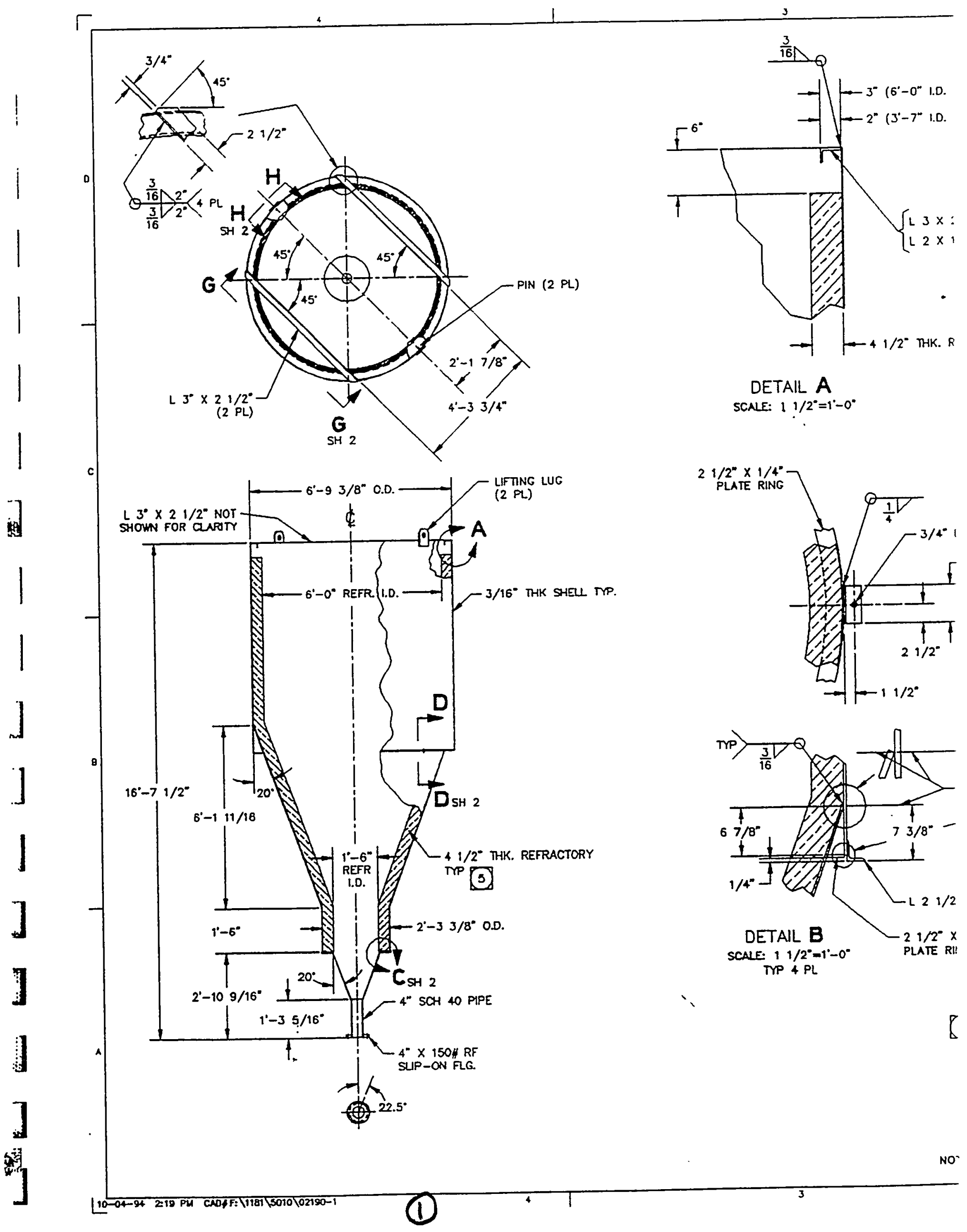




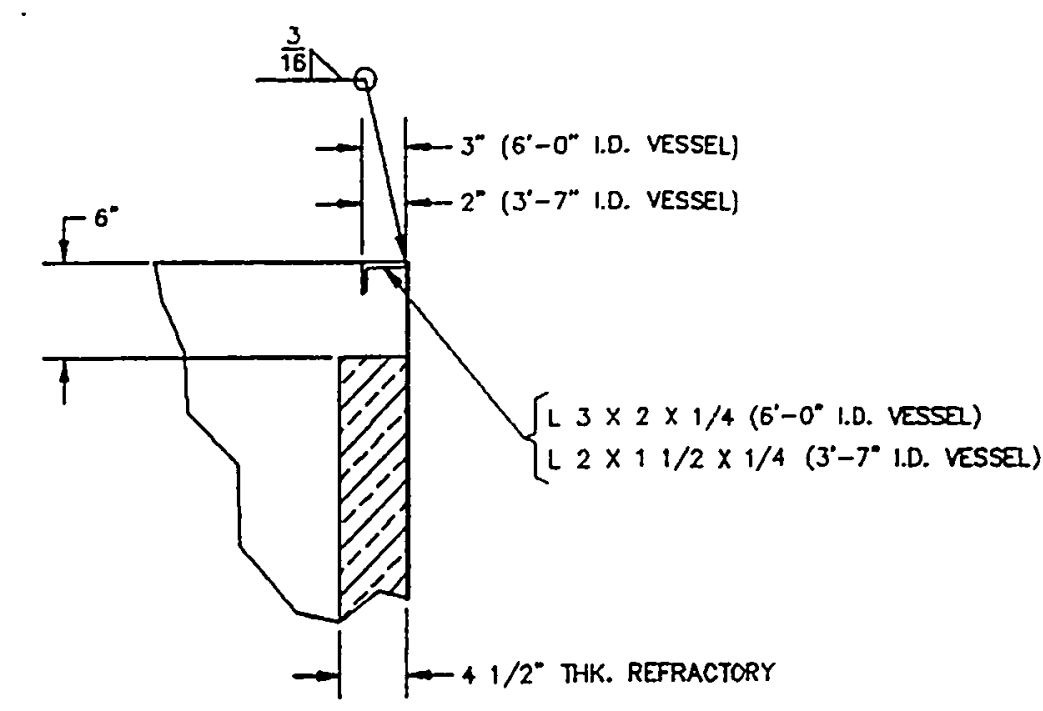

DETAIL $A$

SCALE: $11 / 2^{\circ}=1^{\circ}-0^{\circ}$

$21 / 2^{n} \times 1 / 4^{n}$

PLATE RING
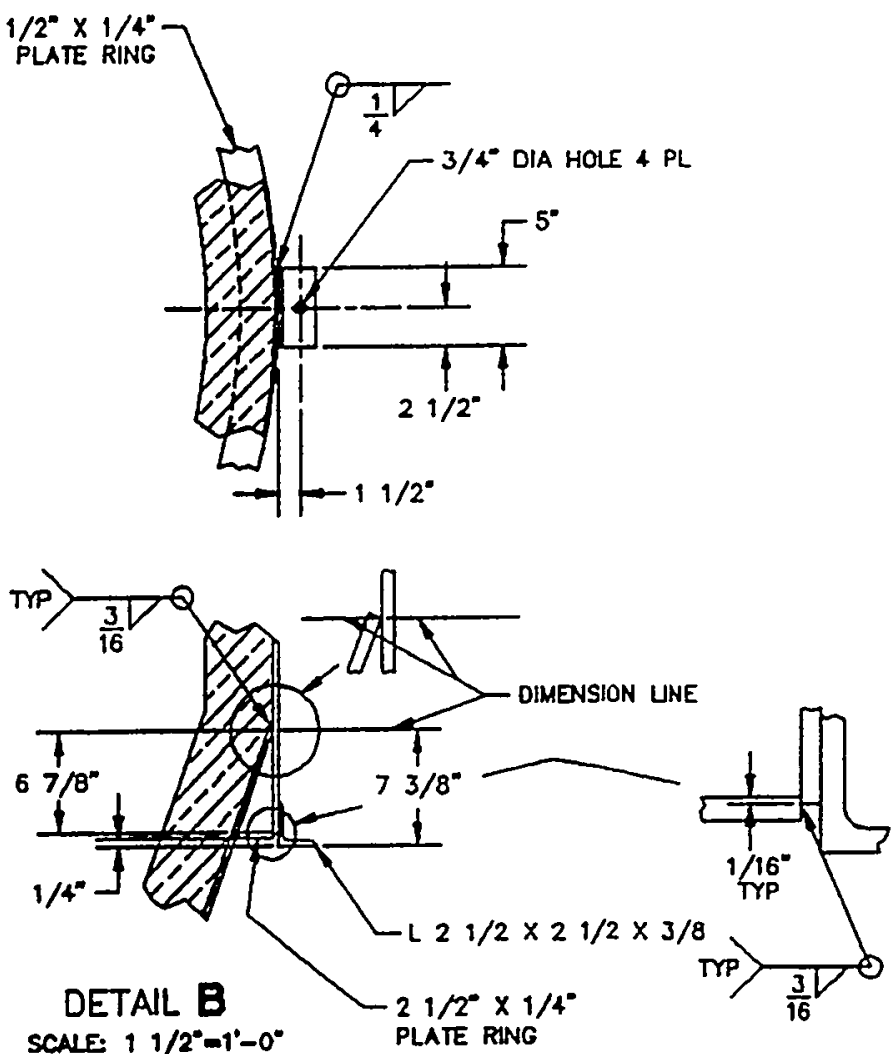

TYP \& PL

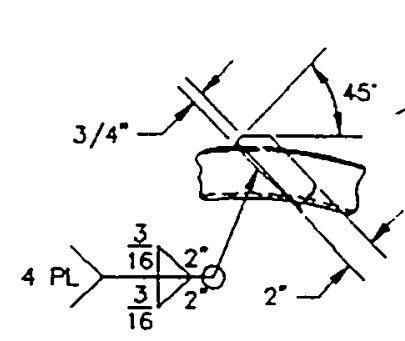

$\mathrm{E}$

(2 PL)

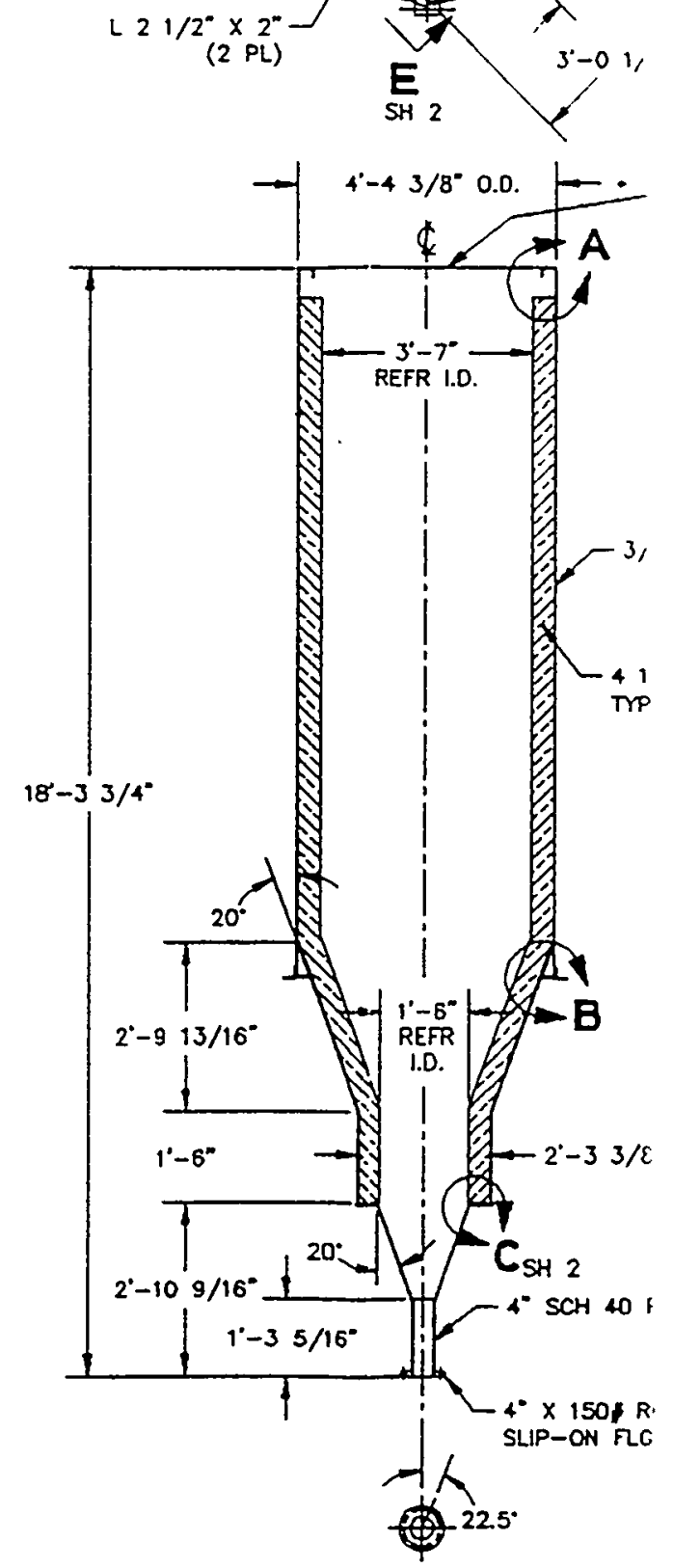

6. BRICK SHEVES MLL BE DEFWND BY REFRACTORY CONTRACTOR.

5 REFPACTORY: SUPER-OUTY BRICK OR EQUAL

4. ALI 3/16" THK CYINDRICAL OR CONICAL PARTS SHALL BE JONED WTH FULL PENETRATION BUTT WELD.

3. BREAK ALI SHARP EDEES

2. ALLOY STEEI FABRICATON PER CPC STII33.

1. AL CARBON STEE SHAPES ND PLATE MATERTAL. PER ASTM A36 OR EQ.

NOTES:

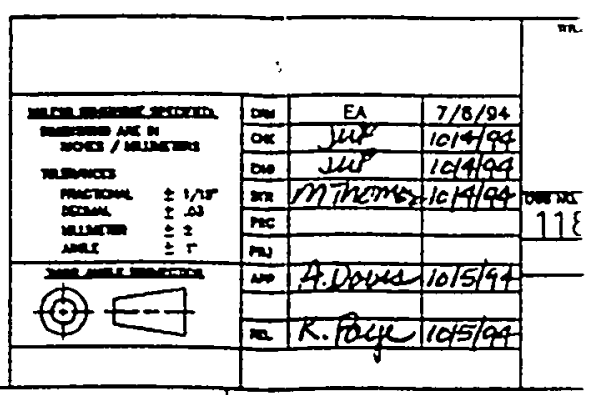

(2) 
isel)

. VESSEl)
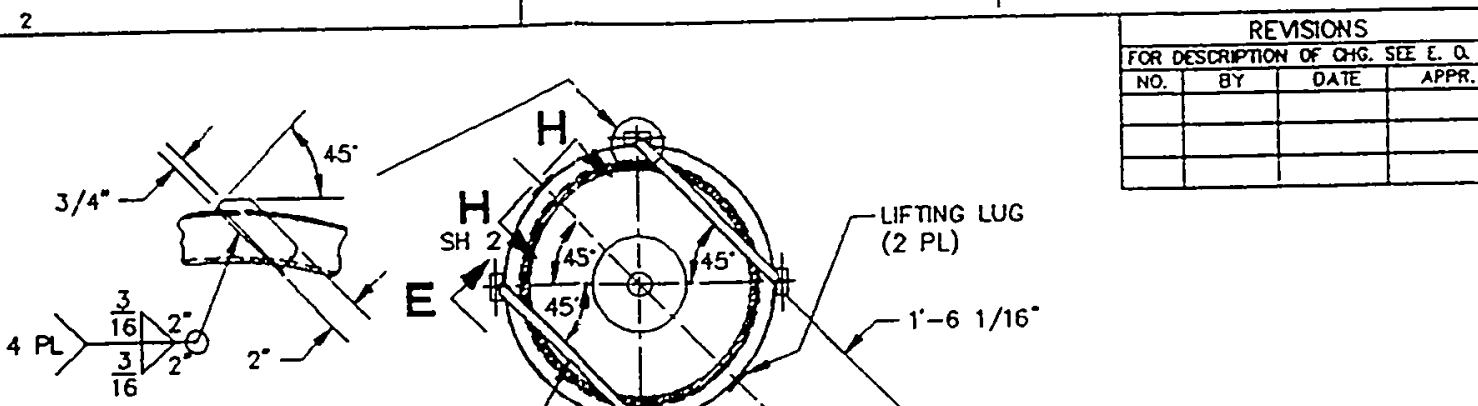

L $21 / 2^{*} \times 2^{n}$

(2 PL)
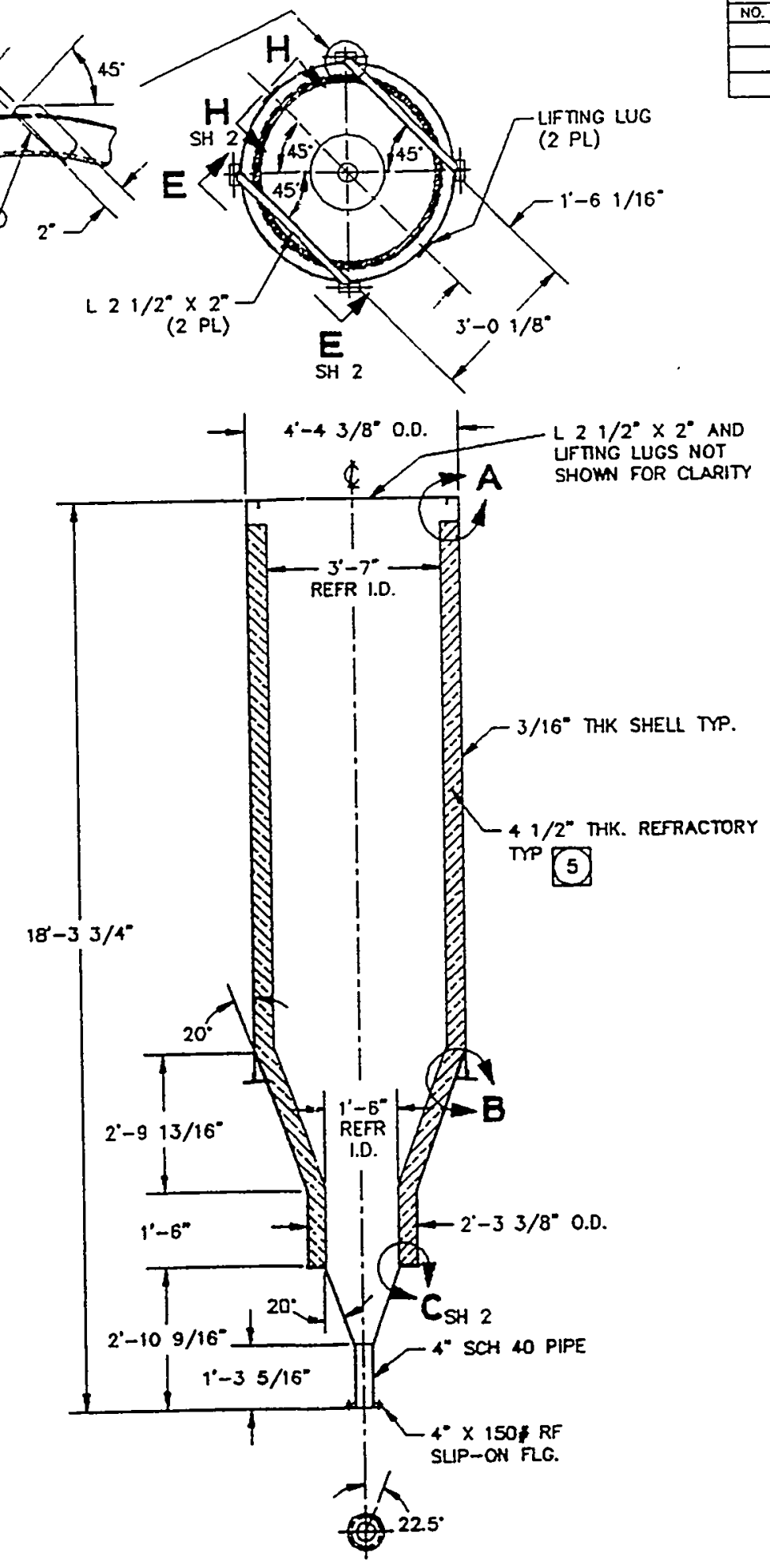

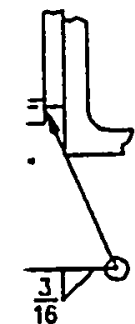

BE DEFNED BY REFRACTORY CONTRACTOR. R DUTY BRICK OR EQUAL. INDRICAL OR CONICAL PARTS TH FUL PENETRATION QUTT MELD. DDGES SATON PER CPC STII33. SHAPES AND PLATE MATEPANL 三Q 


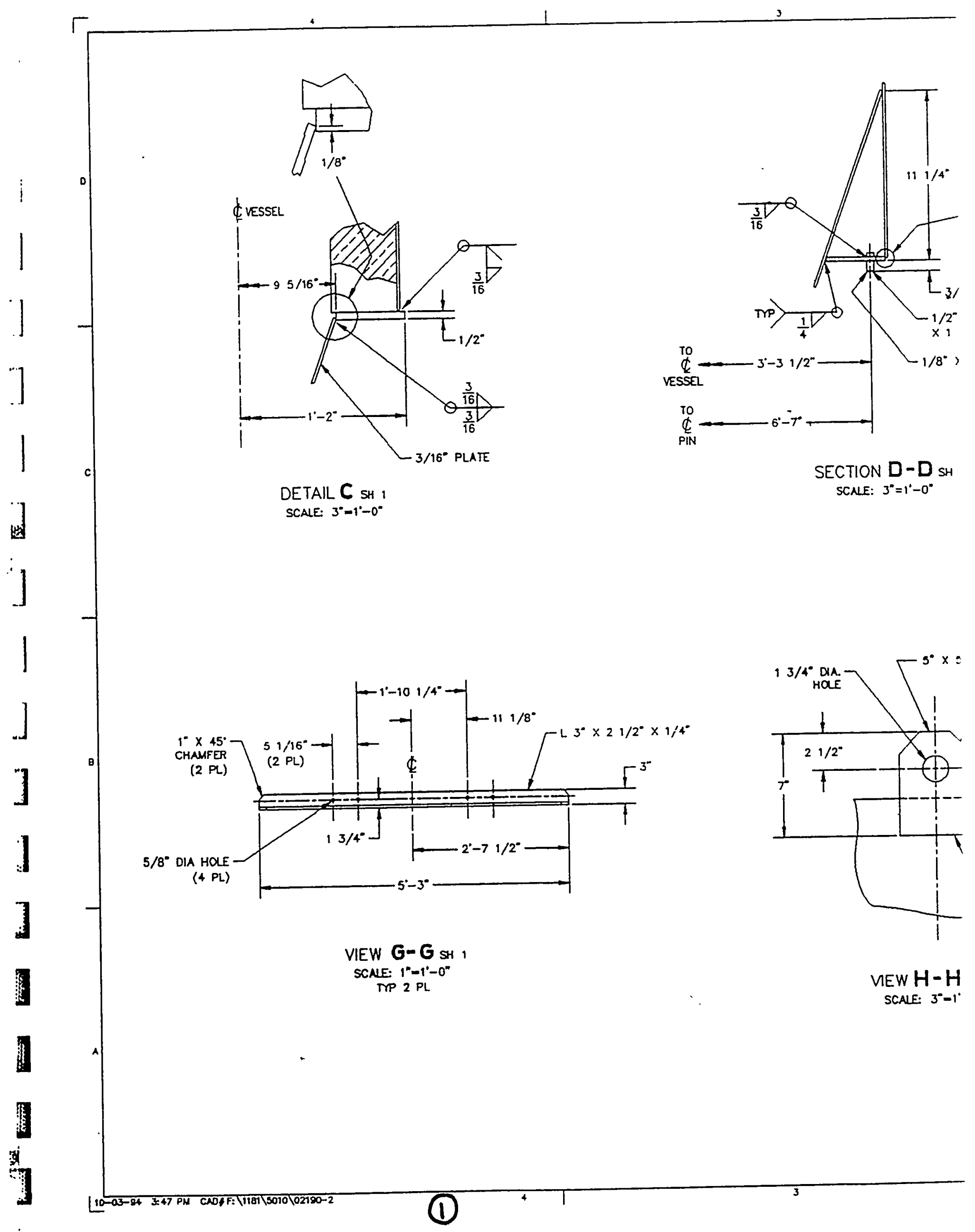



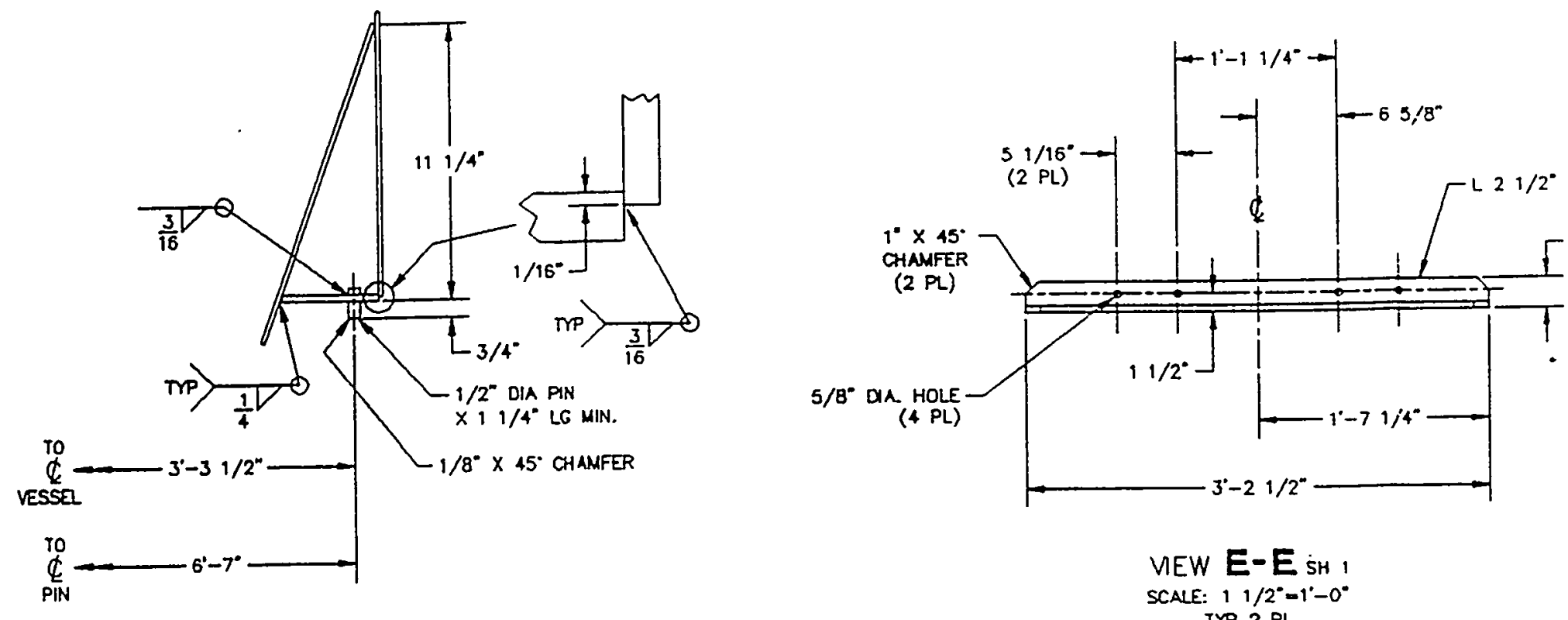

\section{SECTION $\mathbf{D}-\mathbf{D}$ SH 1 \\ SCALE: $3^{n}=1^{\prime}-0^{\circ}$}

VEW E-E $S_{S H} 1$

SCALE: $11 / 2^{\circ}=1^{\circ}-0^{\circ}$ TYP $2 \mathrm{PL}$

$\times 1 / 4^{-}$

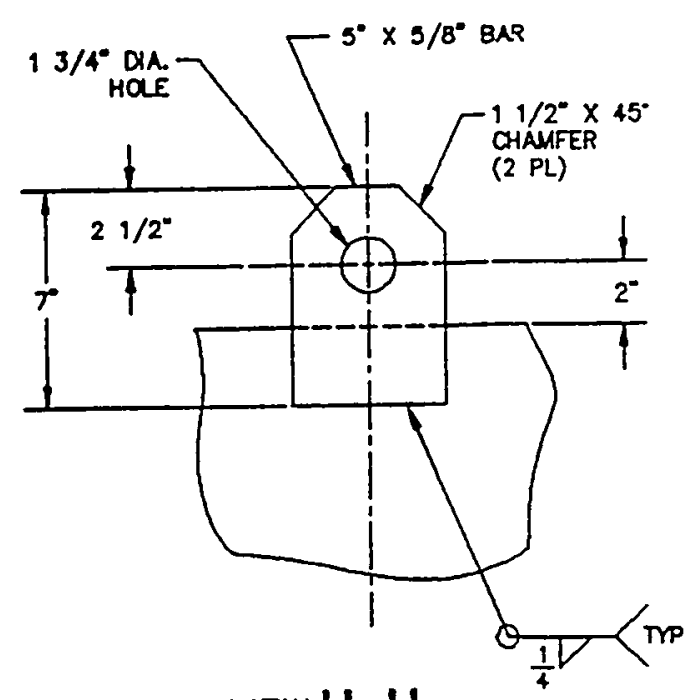

VEW H-H S I

SCALE: $3^{-}-1^{\circ}-0^{*}$ 


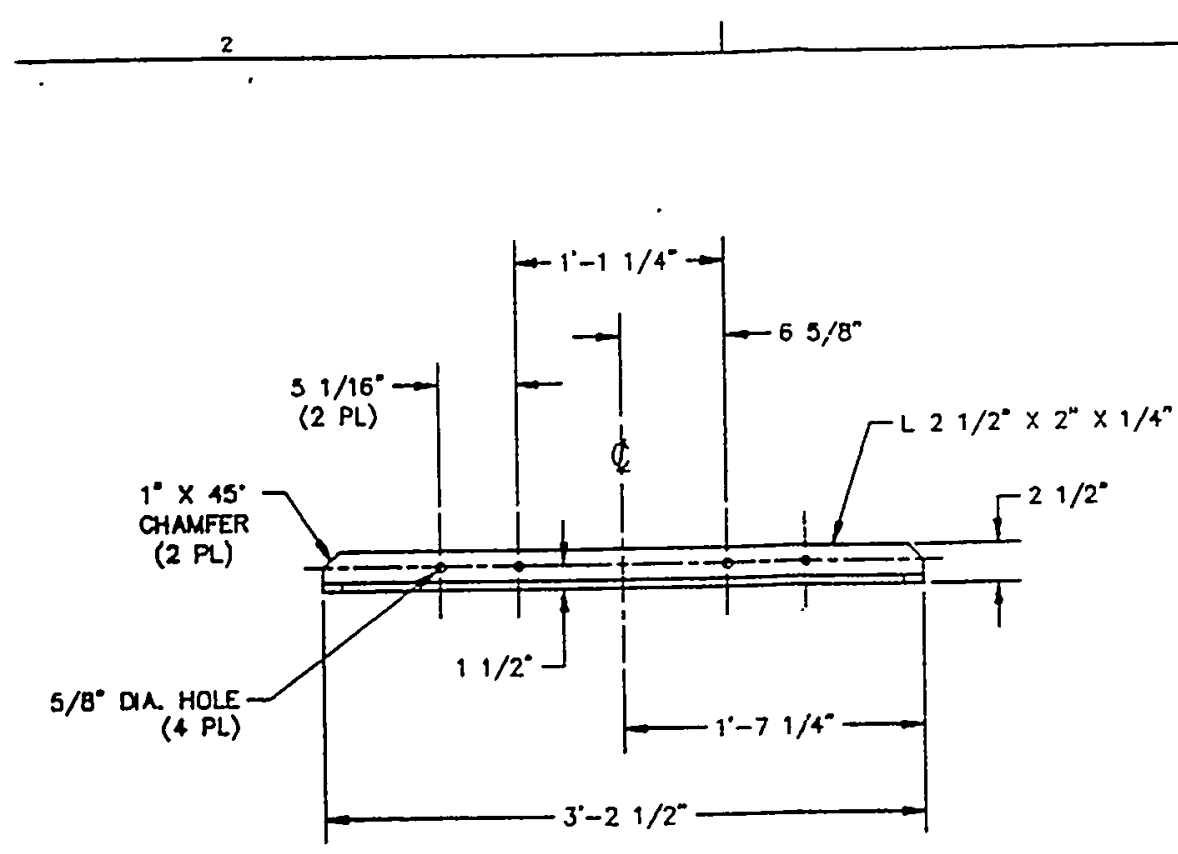

$1+\cdots$

VEW E-E SH 1

SCALE: $11 / 2^{*}=1^{\circ}-0^{\circ}$ TYP $2 \mathrm{PL}$

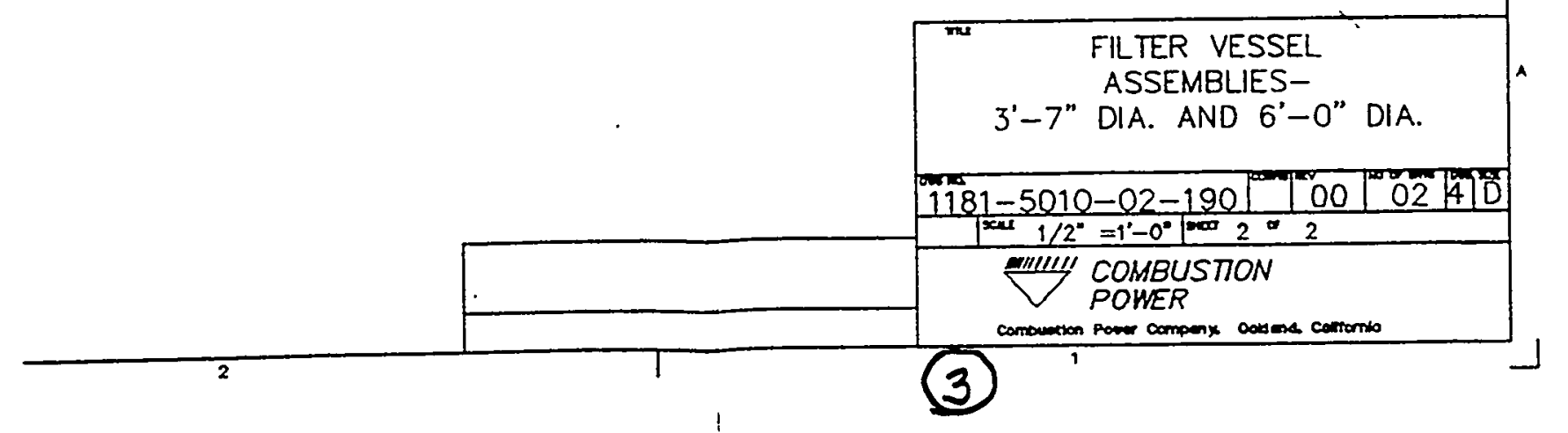



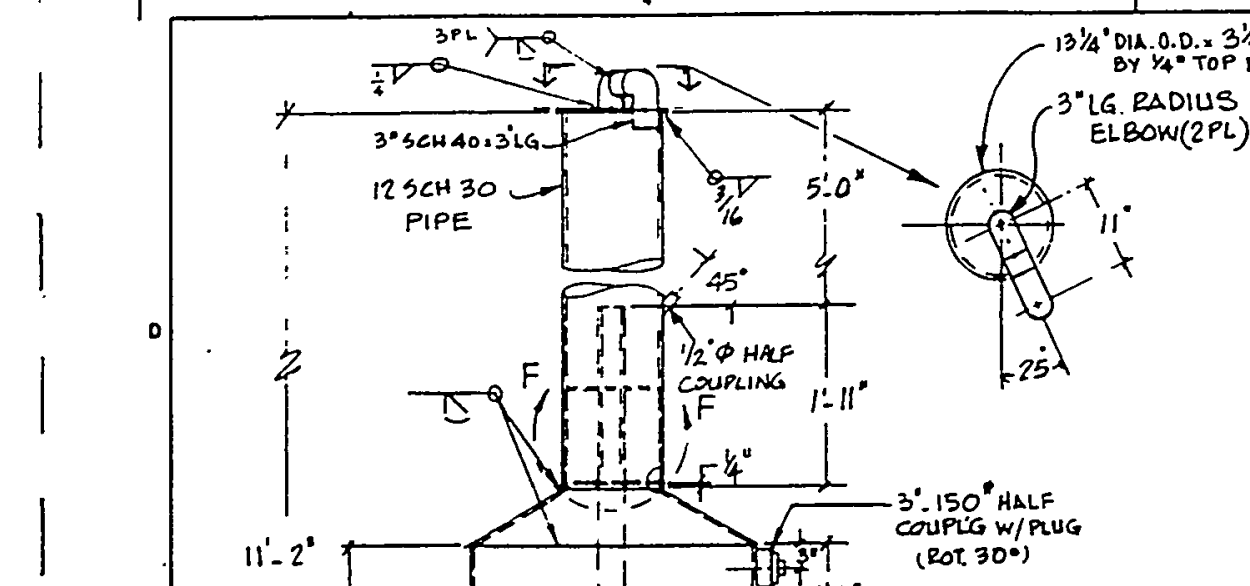

1

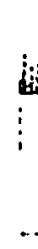

]
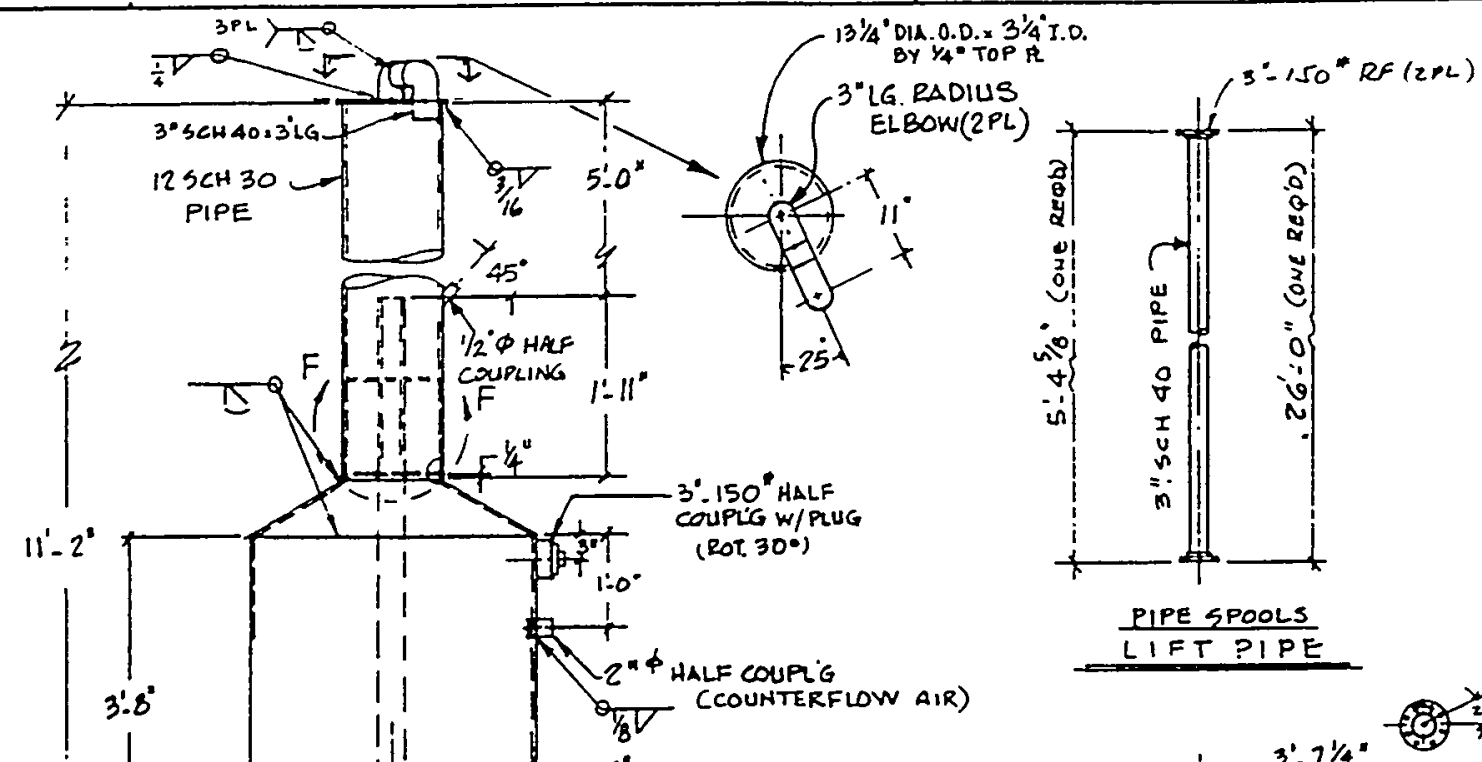

TrP $>-5$

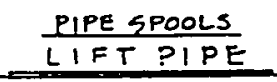

LIFT PIPE
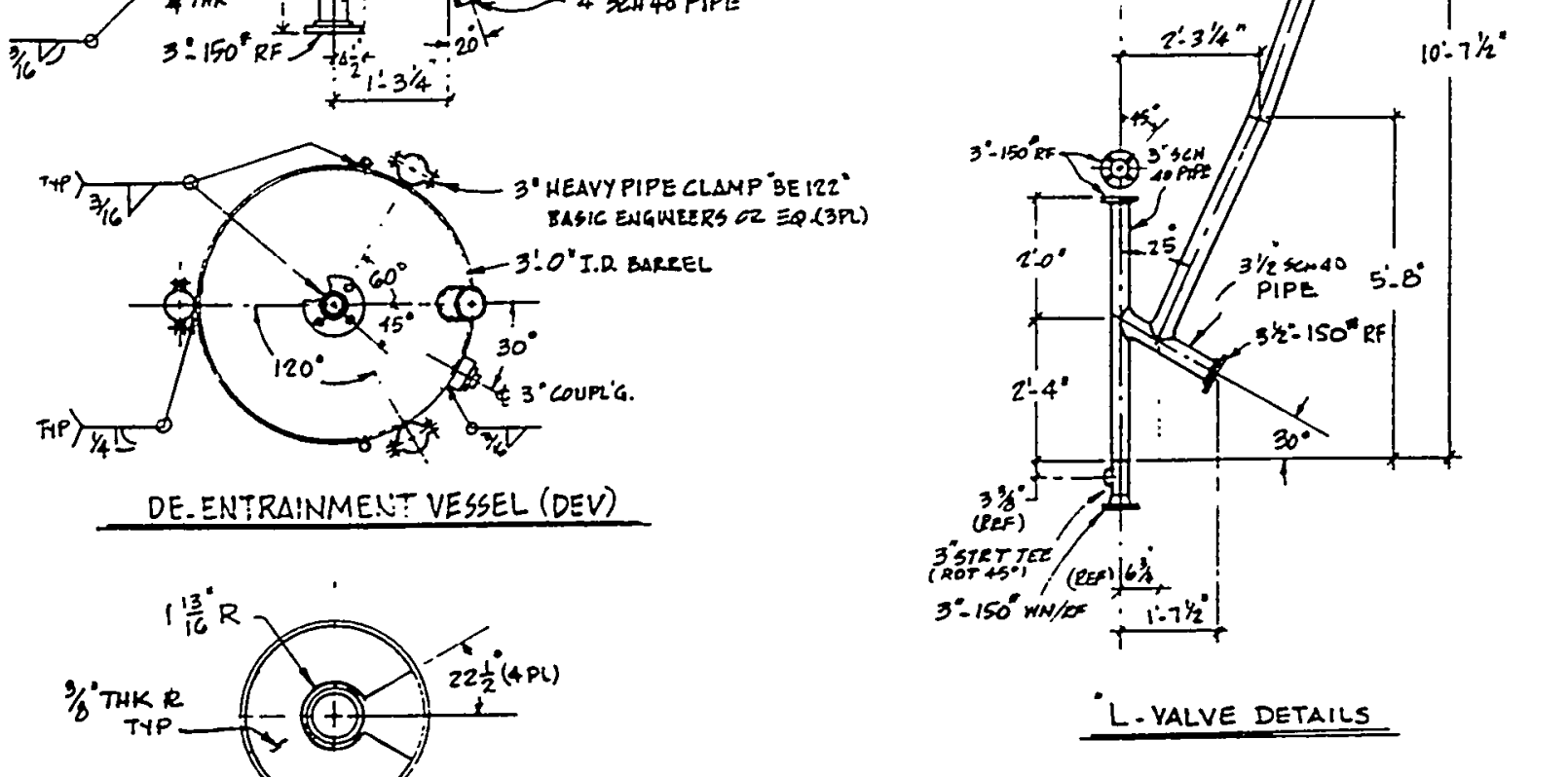

1
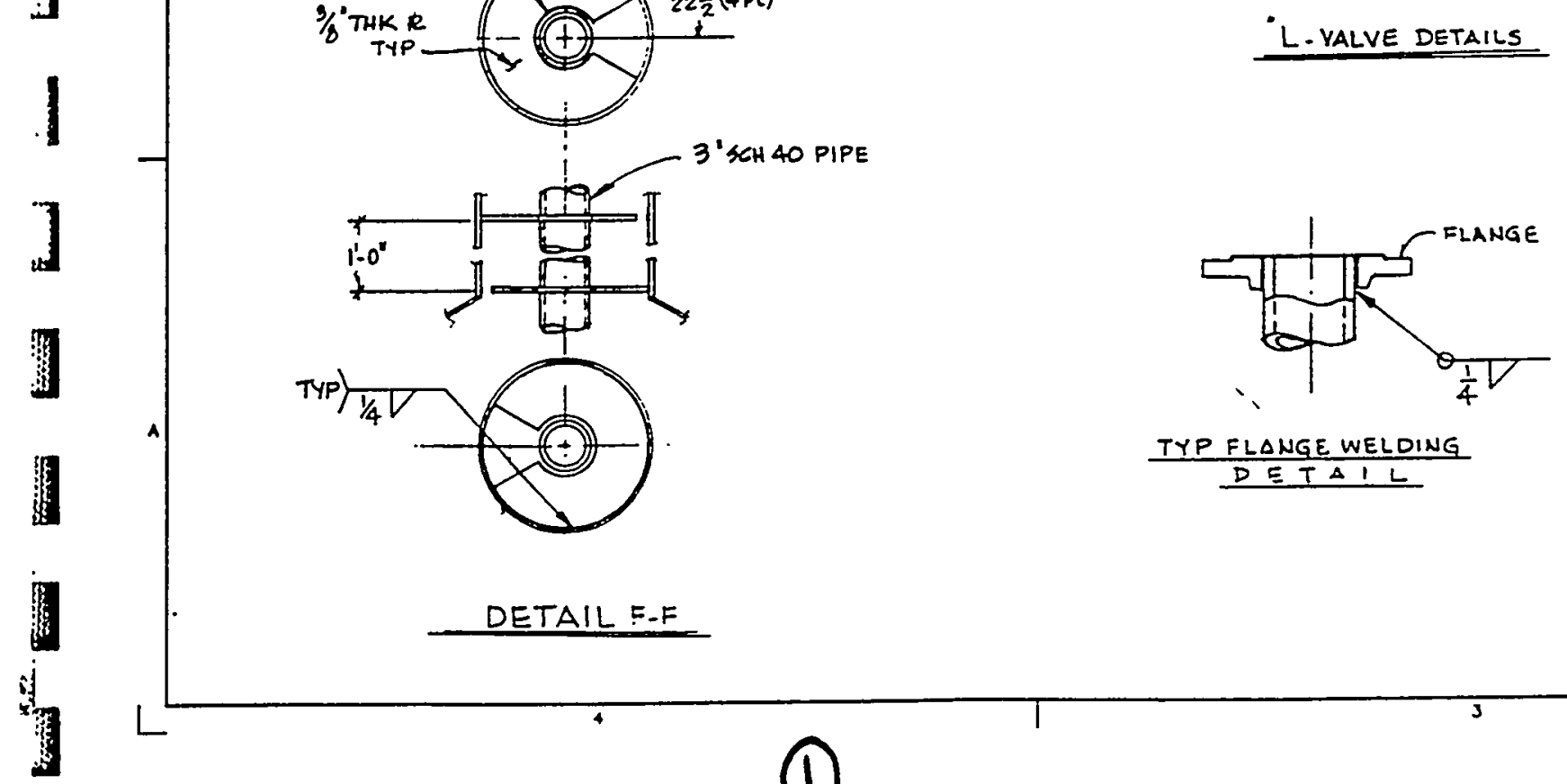

DETAIL F-F

TYP FLANGE WELDING DETAIL 

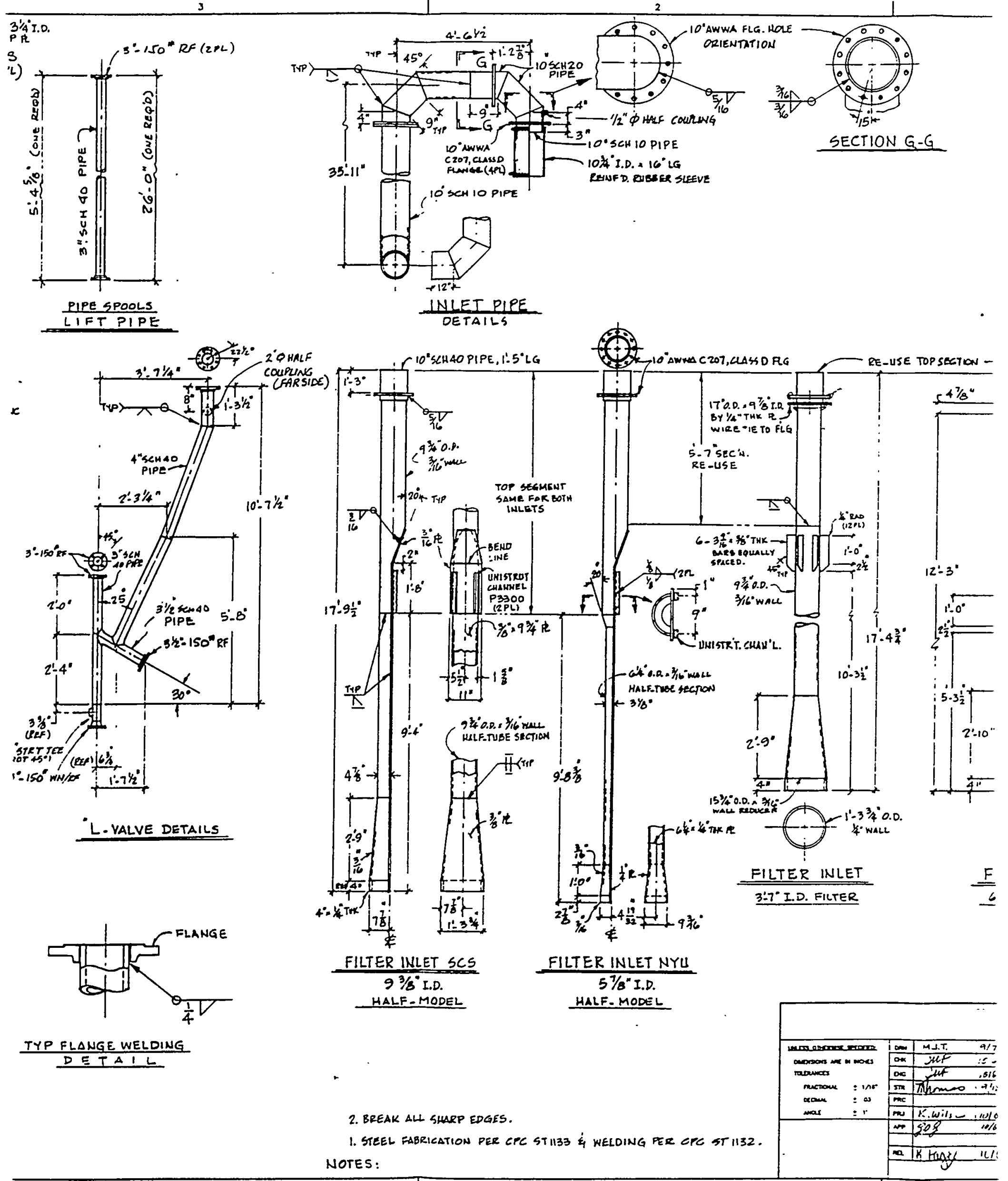


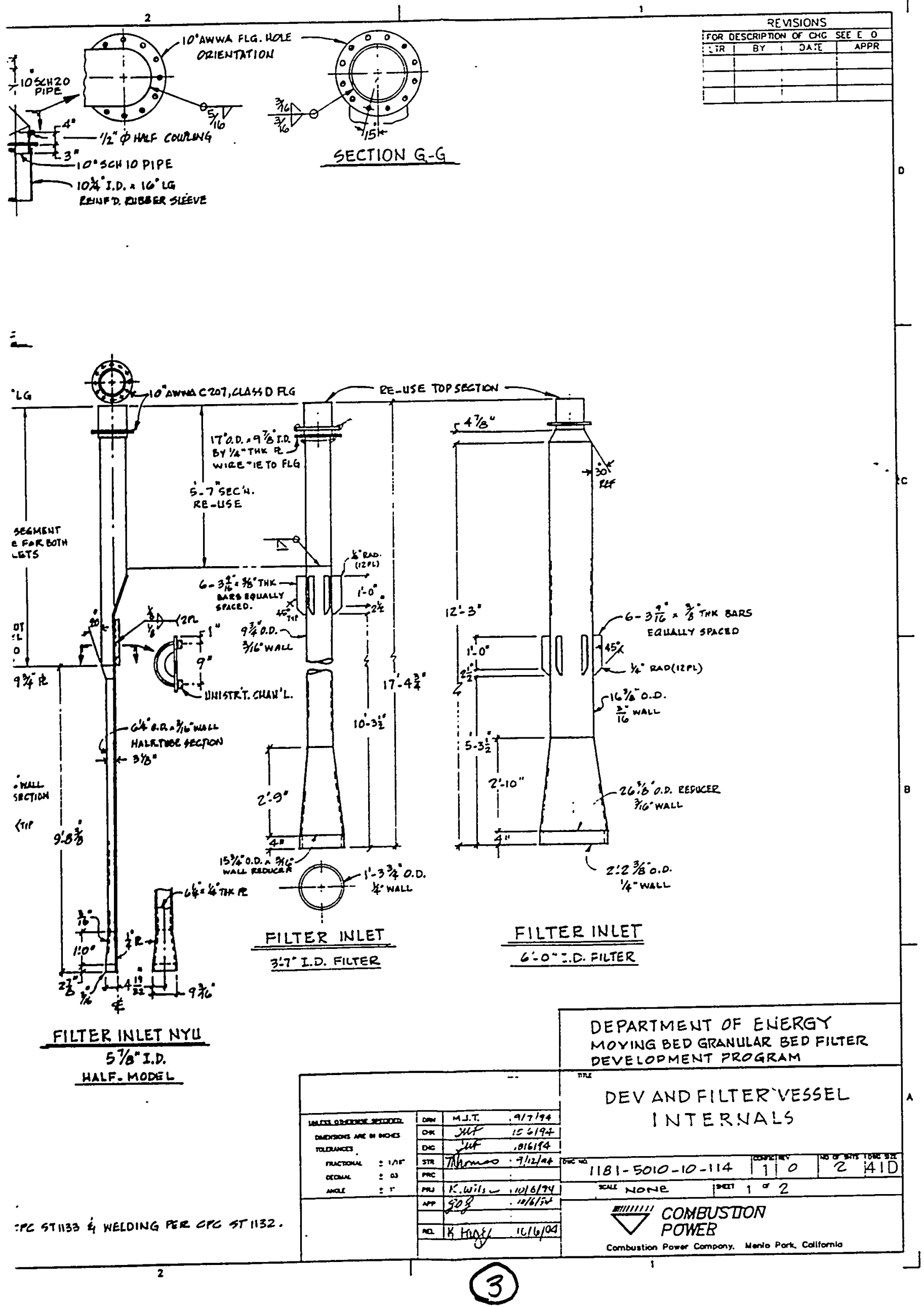




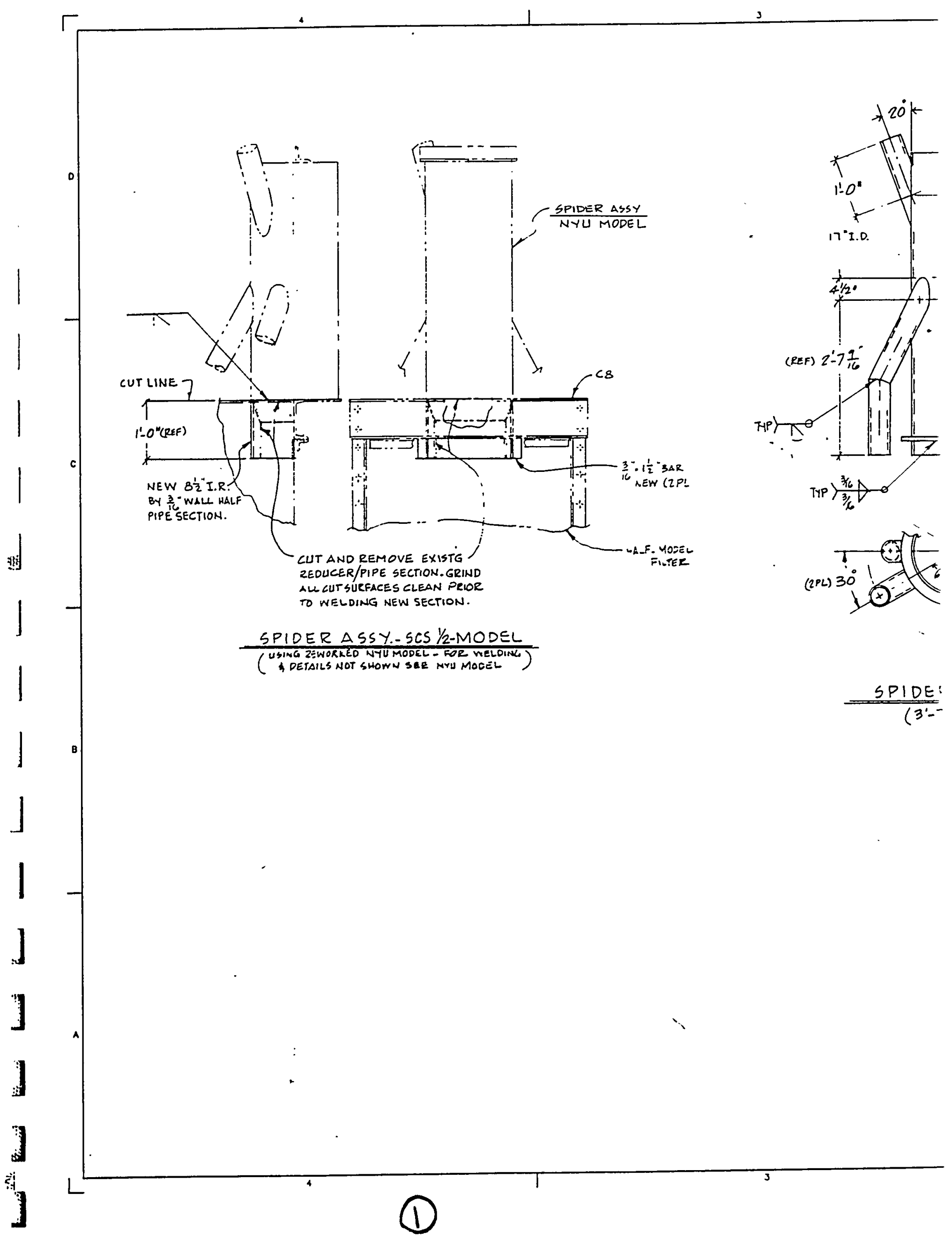




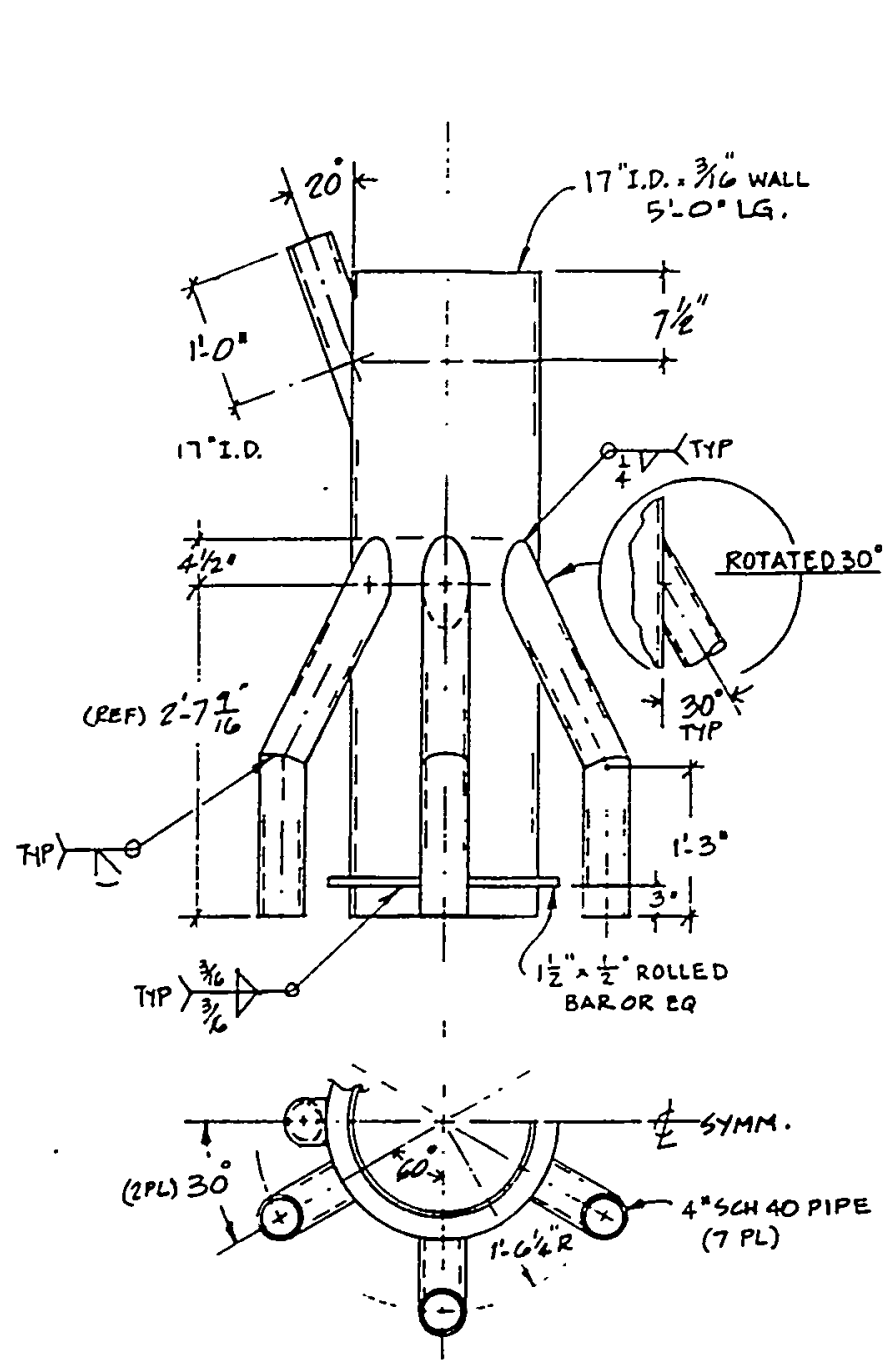

SPIDER ASSY SCS MODEL

(3'-7"=D. FILTER) $\frac{3}{2}: 1 \frac{1}{2} \cdot 3 A R$

NEW (2PL

F. MOJEFirier

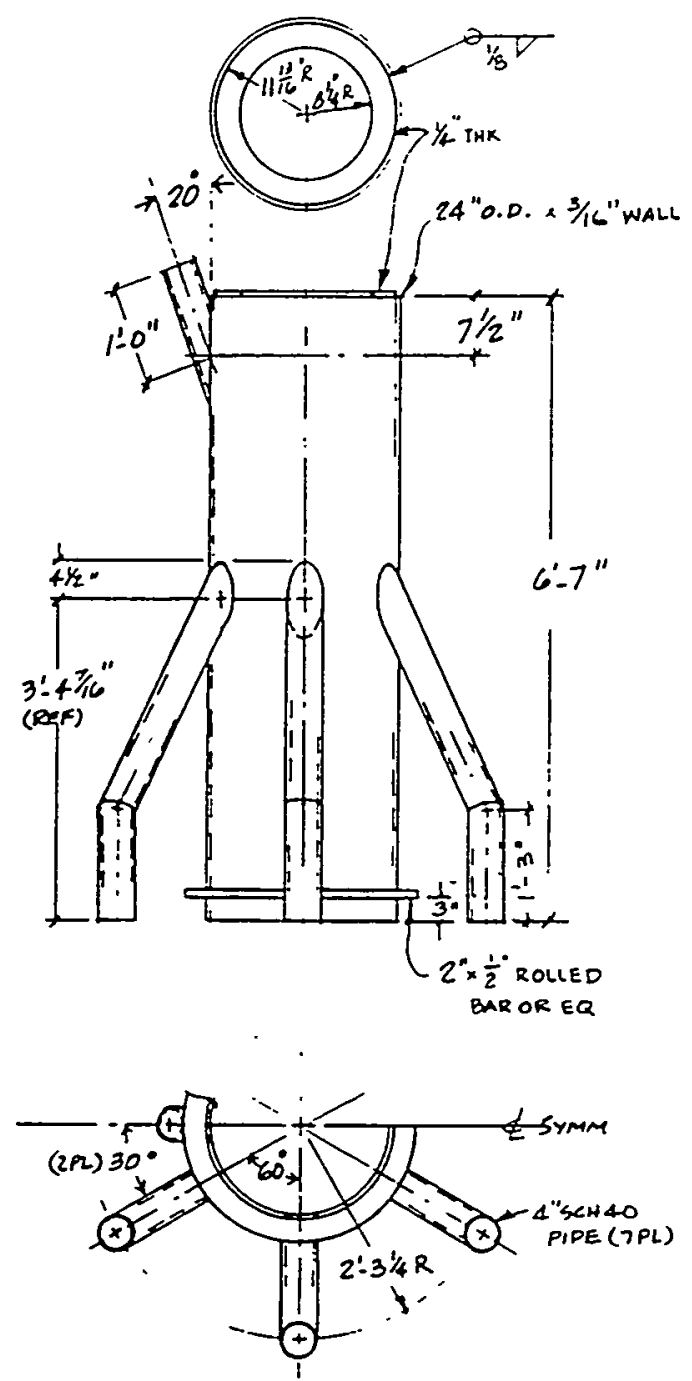

SPIDER ASSY - SCS MODE:(6'-O"I.D. FILTER)

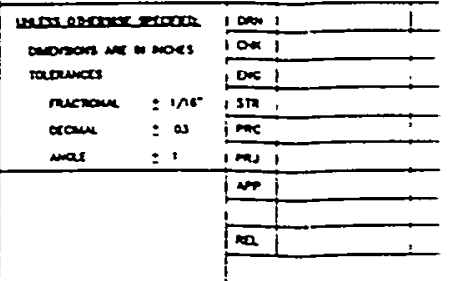




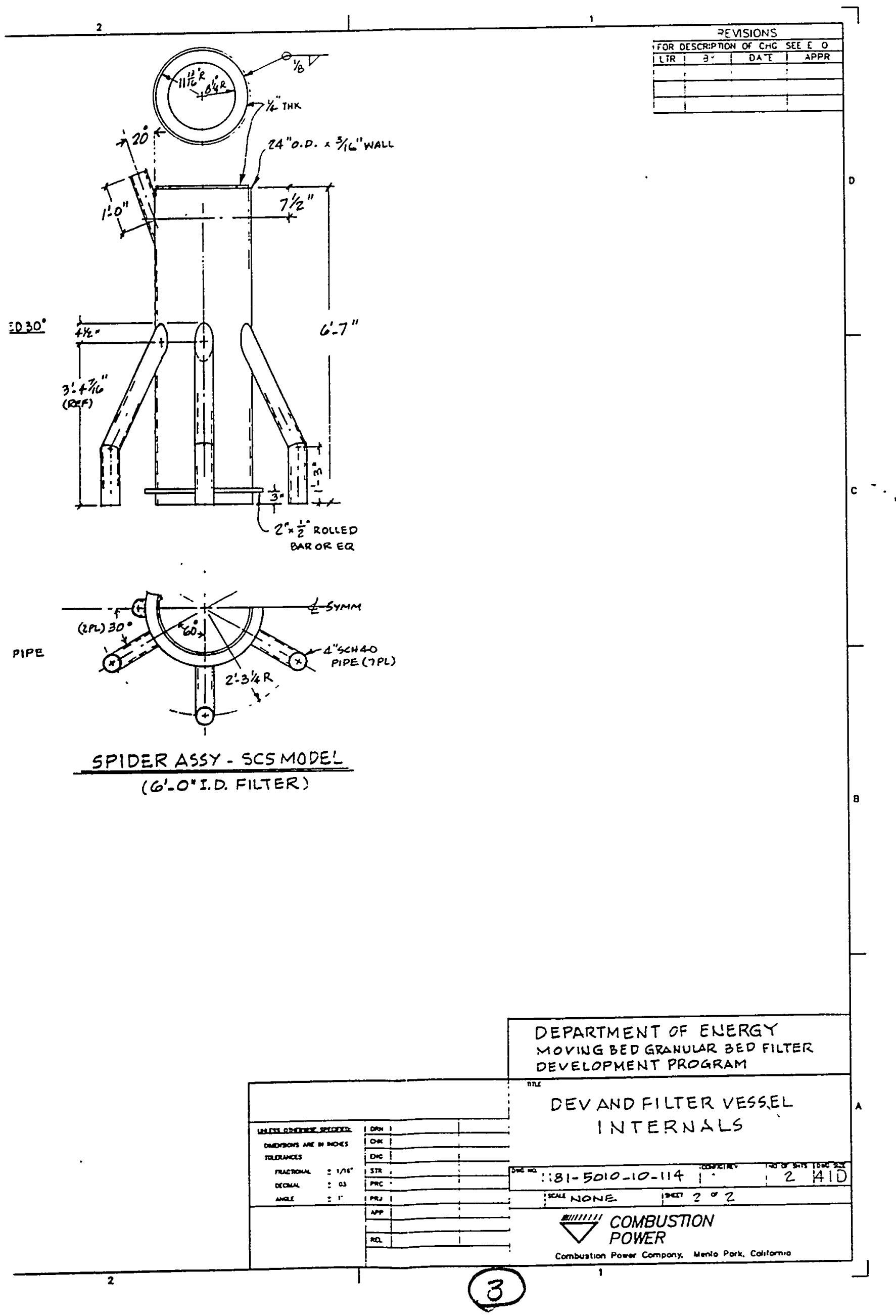




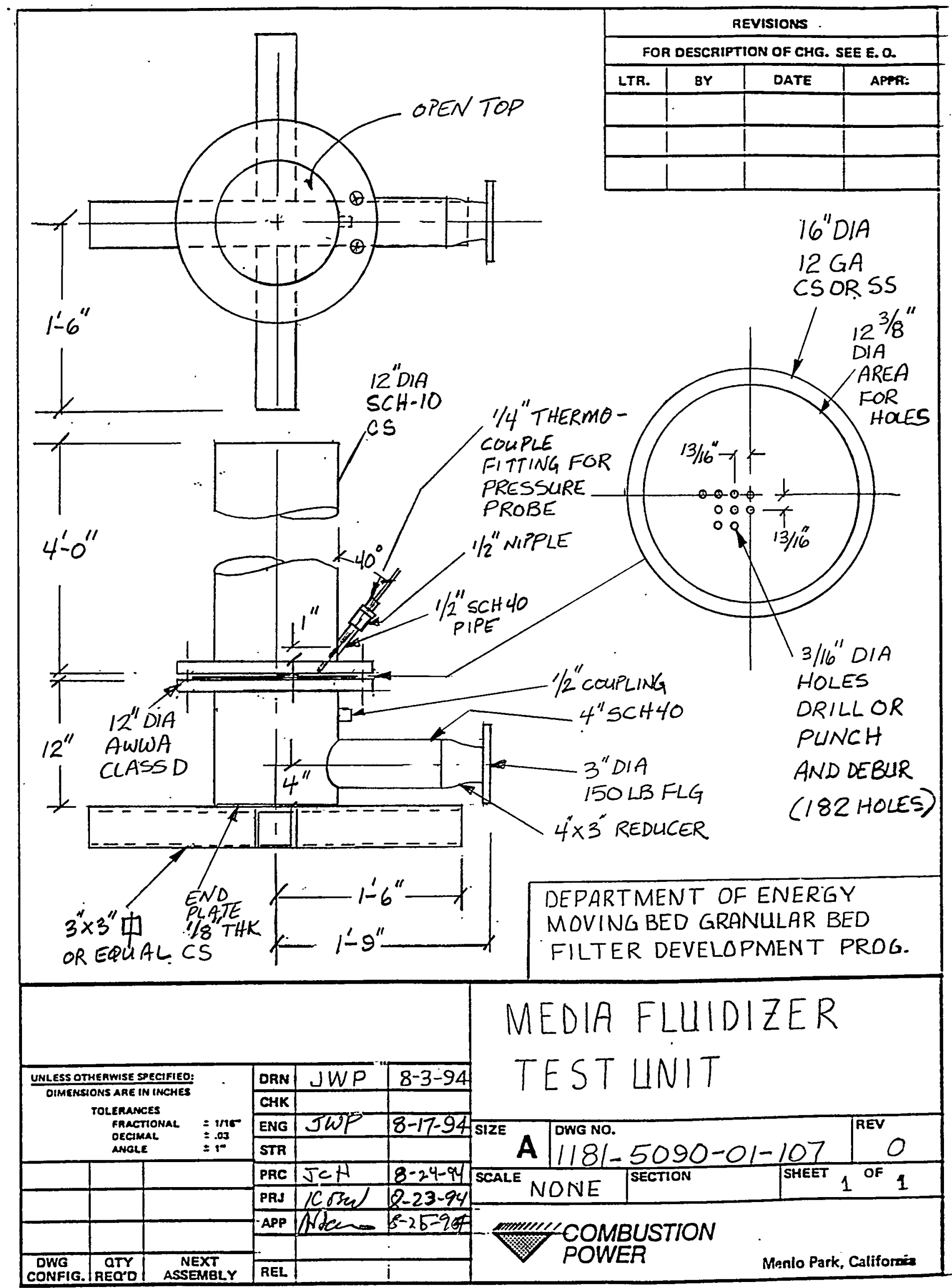



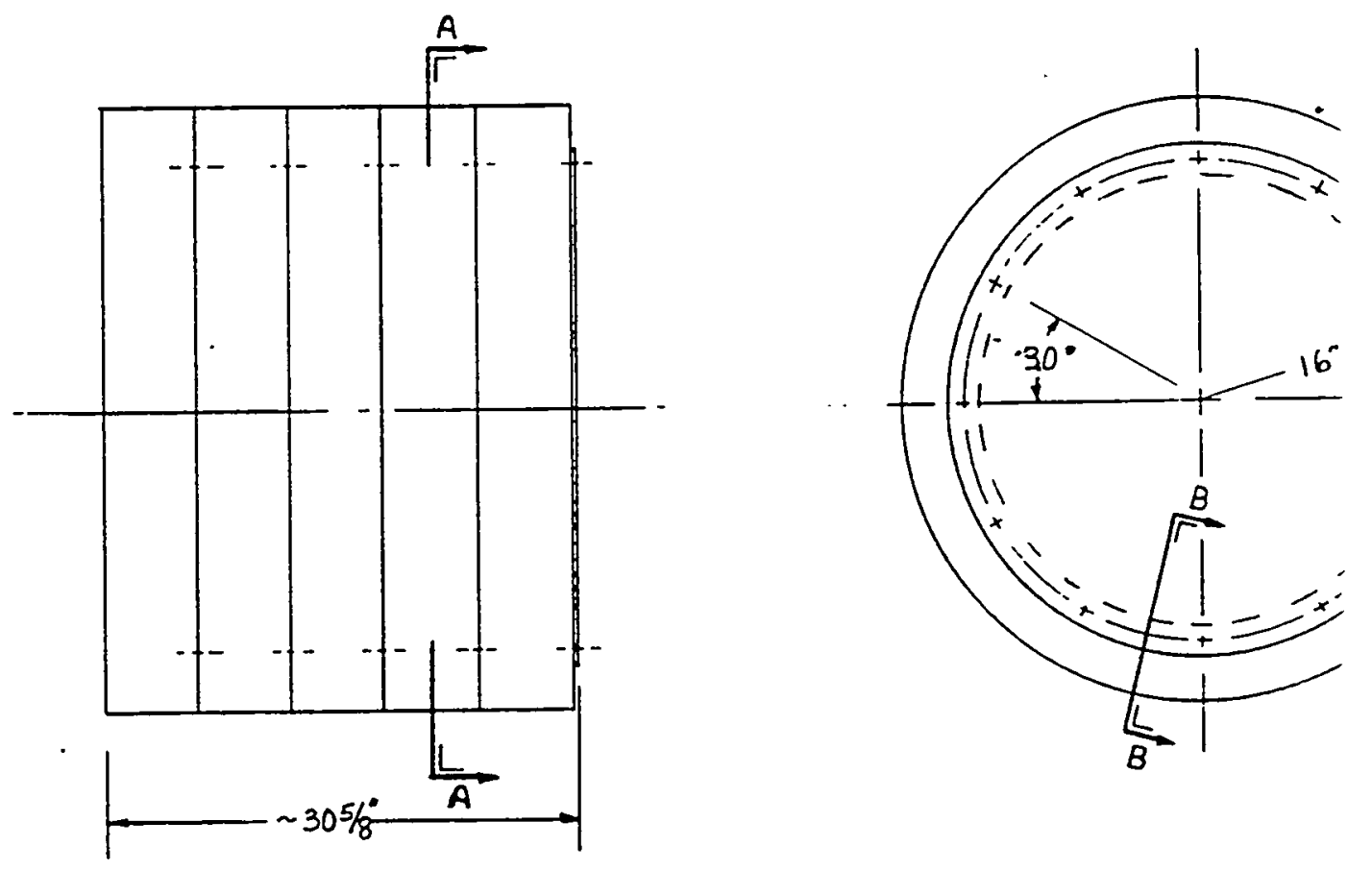

1

D

in

1

1

․

SECTIDN B-B

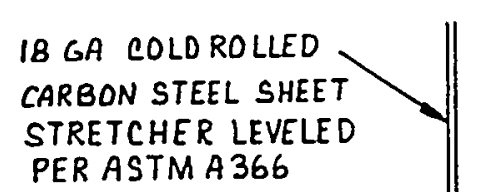

PER ASTM A 366
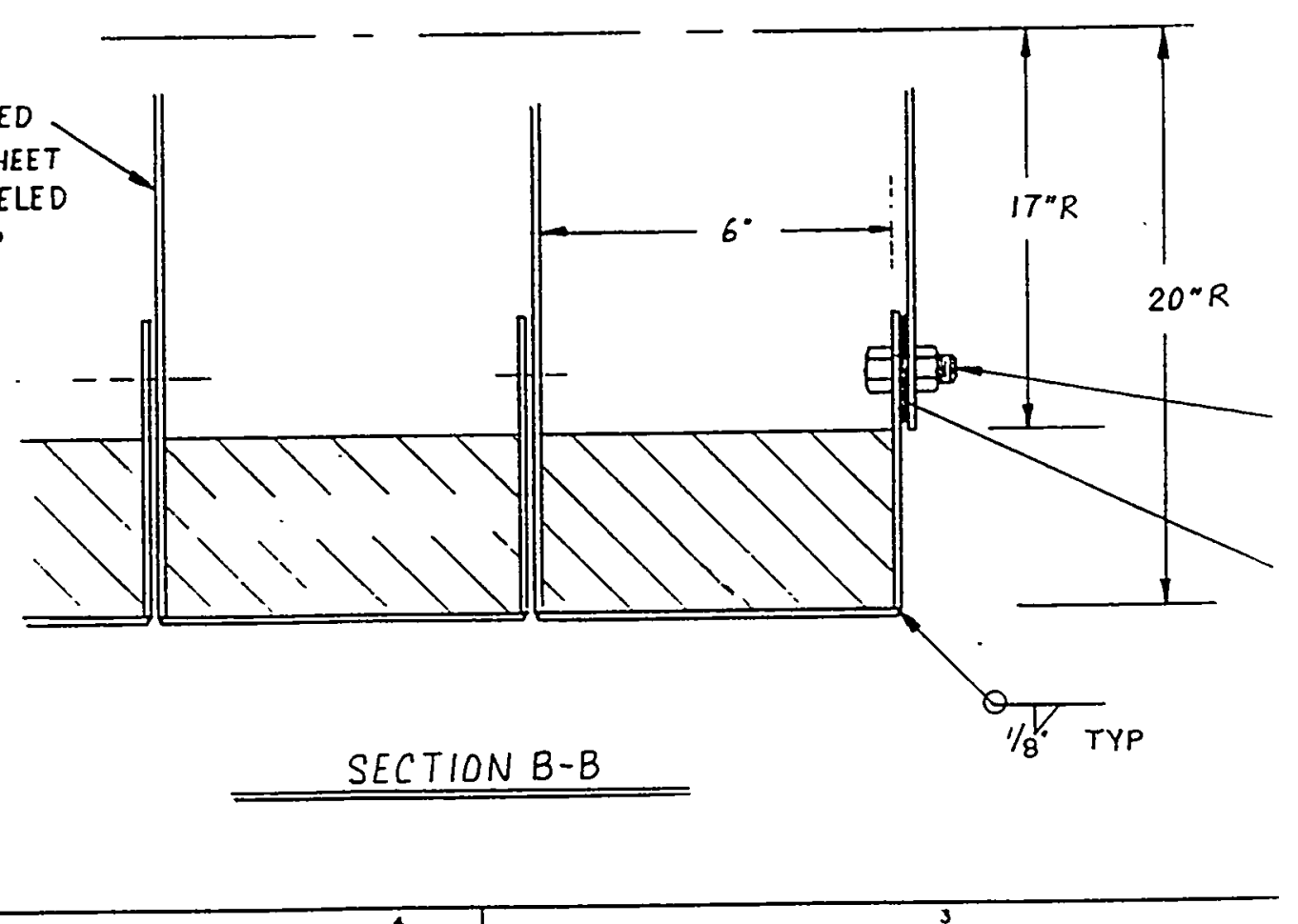

(1)

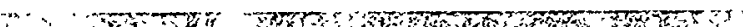



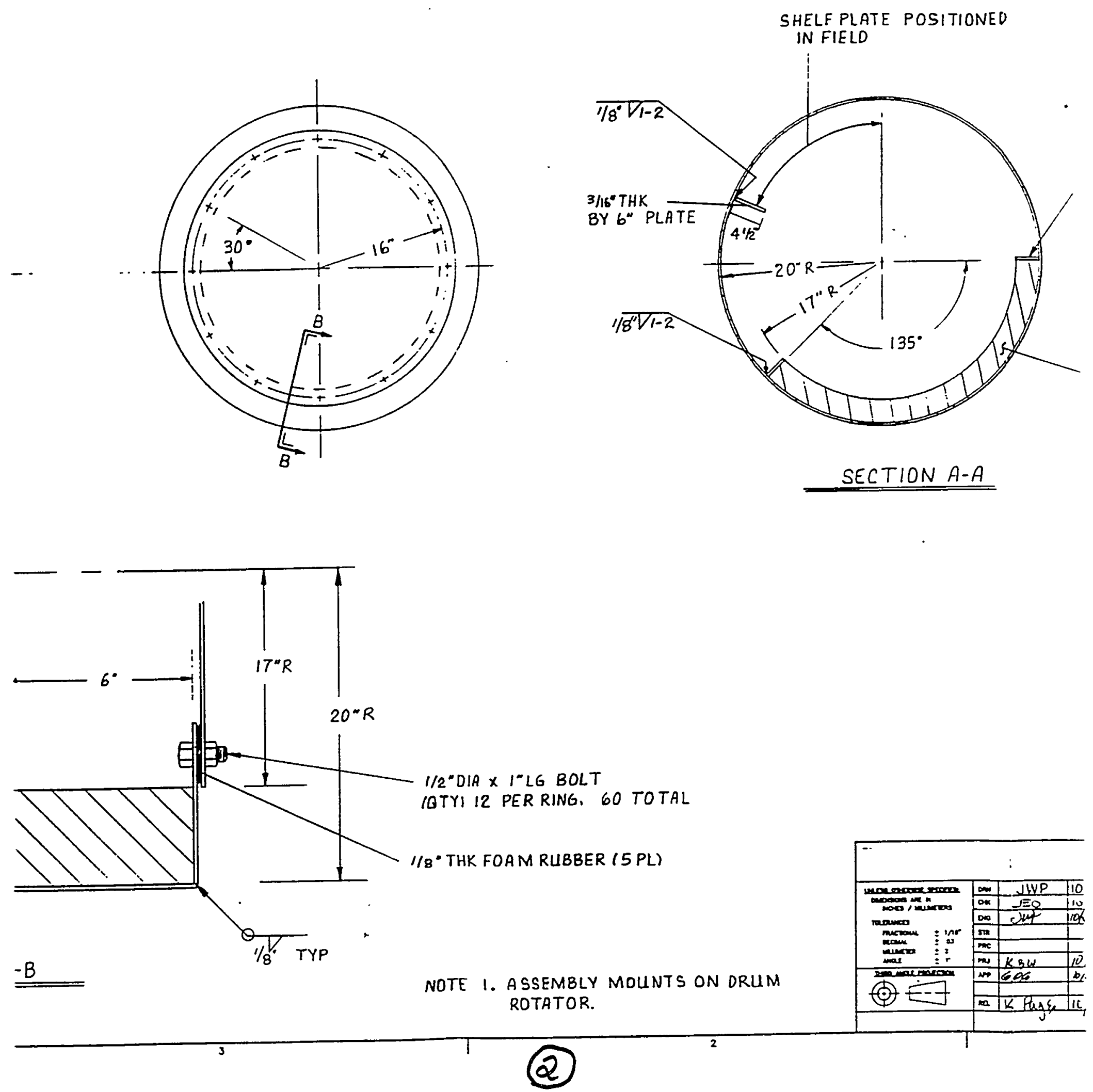

SECTION A-A

$1 / 2$ "DIA $\times 1$ 1"LG BOLT

QTYI 12 PER RING, 60 TOTAL

$18^{\circ}$ THK FOAM RUBBER (5PL)

ROTATOR 


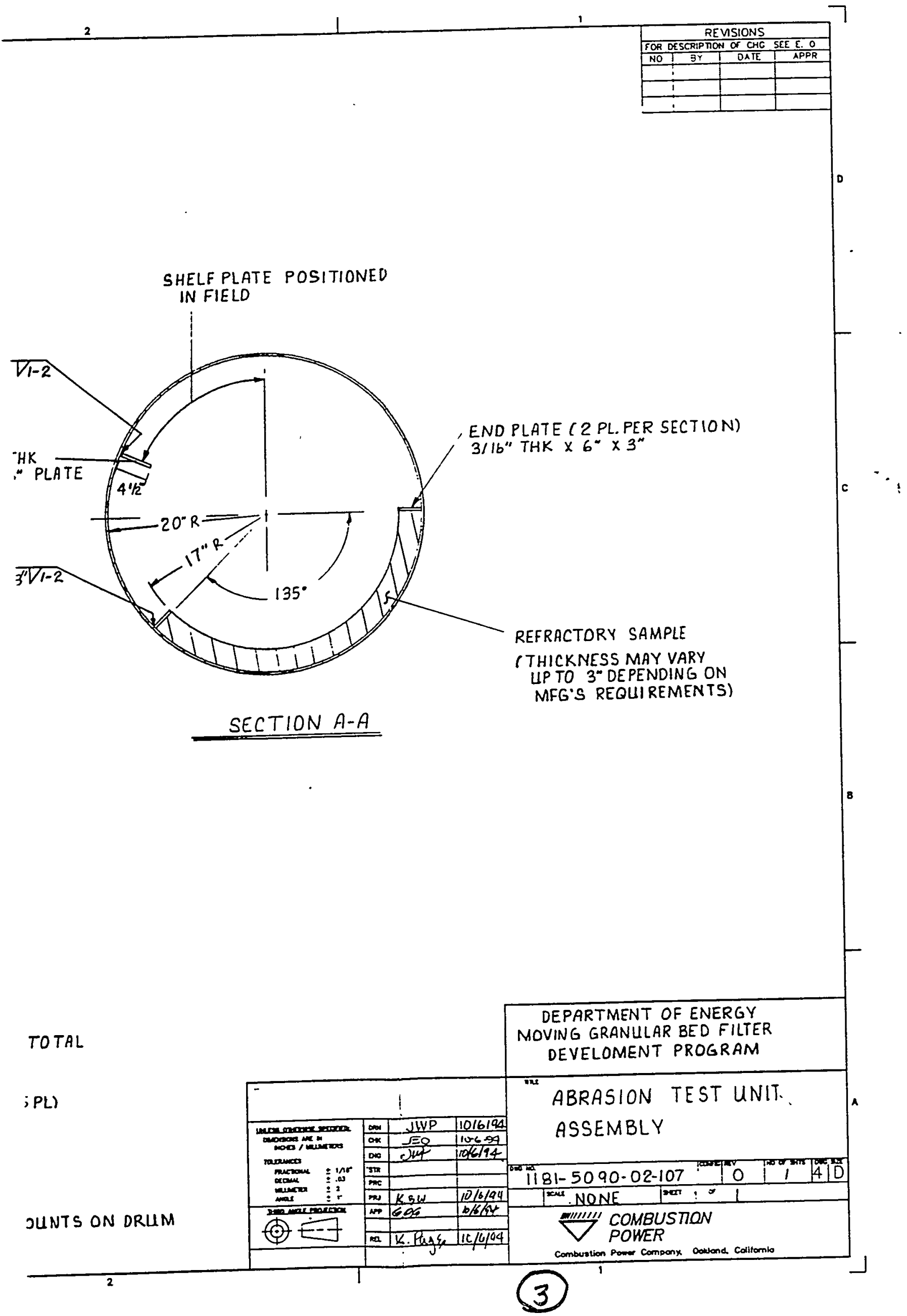

